\title{
OD nanocrystals as light-driven, localized charge injection sources for the contactless manipulation of atomically thin 2D materials
}

\author{
Ilka Kriegel, ${ }^{\mathrm{a}, \mathrm{b}, \mathrm{c}^{*}{ }_{\perp}}$ Michele Ghini, ${ }^{\mathrm{c}, \mathrm{de},{ }_{\perp}}$ Emanuil Sashev Yanev,${ }^{\mathrm{c}}$ Christoph Kastl, ${ }^{\mathrm{f}}$ Kehao Zhang, ${ }^{\mathrm{g}, \mathrm{h}}$ Adam W. Jansons, ${ }^{\mathrm{i}}$ \\ Brandon M. Crockett, ${ }^{\mathrm{i}}$ Kristopher M. Koskela, ${ }^{\mathrm{i}}$ Edward S. Barnard, ${ }^{\mathrm{b}}$ Erika Penzo, ${ }^{\mathrm{b}}$ James E. Hutchison, ${ }^{\mathrm{i}}$ Joshua A. \\ Robinson, ${ }^{\text {g,h }}$ Liberato Manna, ${ }^{\mathrm{e}}$ Nicholas J. Borys, ${ }^{\mathrm{b},{ }^{*}}$, P. James Schuck ${ }^{\mathrm{c}^{*}}$ \\ ${ }^{a}$ Functional Nanosystems, Istituto Italiano di Tecnologia (IIT), via Morego 30, I-16163 Genova, Italy \\ ${ }^{\mathrm{b}}$ The Molecular Foundry, Lawrence Berkeley National Laboratory, Berkeley, CA, USA \\ ${ }^{\mathrm{c}}$ Department of Mechanical Engineering, Columbia University, New York, NY, USA \\ ${ }^{\mathrm{d}}$ Dipartimento di Chimica e Chimica Industriale, Università degli Studi di Genova, Via Dodecaneso 31, 16146 Genova, \\ Italy \\ ${ }^{e}$ Department of Nanochemistry, Istituto Italiano di Tecnologia (IIT), via Morego 30, I-16163 Genova, Italy \\ ${ }^{\mathrm{f}}$ Walter Schottky Institute and Physics Department, Technical University of Munich, Am Coulombwall 4a, 85748 \\ Garching, Germany \\ ${ }^{\mathrm{g}}$ Department of Materials Science and Engineering, Center for 2-Dimensional and Layered Materials, The Pennsylvania \\ State University, University Park, Pennsylvania, 16802, United States \\ ${ }^{\mathrm{h}}$ Center for Atomically Thin Multifunctional Coatings (ATOMIC), The Pennsylvania State University, University Park, \\ Pennsylvania, 16802, United States \\ ${ }^{\mathrm{i}}$ Materials Science Institute and Department of Chemistry, University of Oregon, Eugene, Oregon 97403, United States \\ ${ }^{j}$ Department of Physics, Montana State University, Bozeman, MT, USA \\ ${ }^{\perp}$ These authors contributed equally to this work.
}

\section{Abstract:}

We report a new localized and electrodeless charge injection scheme that quasi-permanently modifies monolayer (1L-) $\mathrm{MoS}_{2}$ doping levels to extents competing with electrostatic gating. The key innovation is to use Sn-doped $\mathrm{In}_{2} \mathrm{O}_{3}$ (ITO) nanocrystals (NCs) as contactless light-driven charge injection sources triggered solely by light. Each nanocrystal can store and transfer multiple charges after ultraviolet illumination within the diffraction limited laser spot. This results in reductions in carrier density in the underlying $1 \mathrm{~L}-\mathrm{MoS}_{2}$ up to $1 \times 10^{13} \mathrm{~cm}^{-2}$ and is observed throughout the extent of the $2 \mathrm{D}$ material flake. The long-distance charge separation proliferates up to $40 \mu \mathrm{m}$ away from the localized charge injection and persists over months. The apparent driving force for carrier relocation is the initial inhomogeneous electronic landscape of the 2D material. These studies demonstrate a novel all-optically controlled tool to locally inject carriers with sub-micrometer precision. This new ability allows us to extract important aspects of inhomogeneity in 2D materials undisturbed by bulky electronic contacts and indicates that local 2D material manipulation can serve as a key element for novel nanoscale device design. 


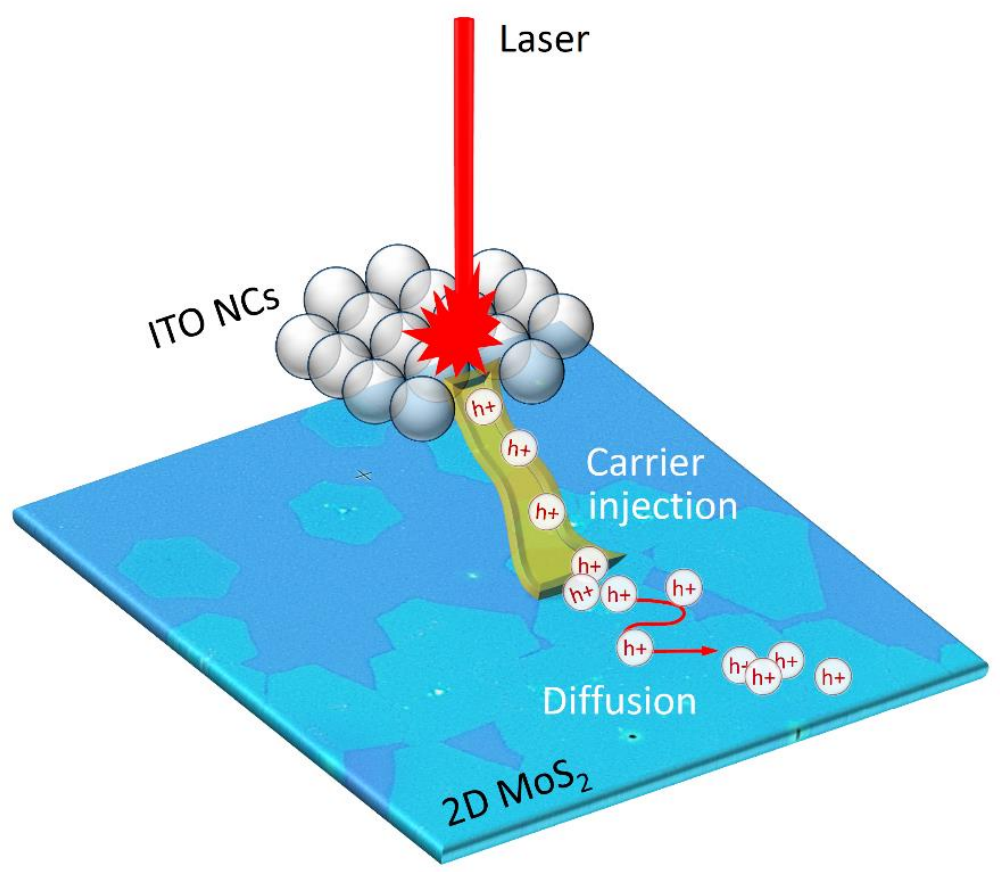

TOC: Illustration of the light-driven localized charge injection followed by carrier diffusion

\section{Introduction:}

Two dimensional transition metal dichalcogenides (2D TMDCs) have exceptional electronic, optical, mechanical, chemical, and thermal properties. ${ }^{1-3}$ Due to their monolayer nature, their electronic structure and optoelectronic properties are exquisitely sensitive to external stimuli, such as electrostatic interactions with the substrate, ${ }^{4}$ defects, ${ }^{5-7}$ vacancies, ${ }^{8}$ strain, ${ }^{9,10}$ or doping, ${ }^{11}$ providing local variation of their optoelectronic properties. ${ }^{4,12}$ Recently, local manipulation has been positively implemented to design novel device architectures, ranging from energy funnels for solar energy conversion ${ }^{9,12,13}$ to quantum optics. ${ }^{14}$ At the same time, this sensitivity to the local surrounding can dramatically impact their overall optoelectronic response. Notably, 2D TMDC-based devices require the connection to metal contacts, that can invasively impact and negatively perturb their local properties resulting in an overall reduction of performance. ${ }^{16}$

Light is an attractive tool to locally modulate the optoelectronic properties of 2D materials in a contactless way. When excited with light, temporary changes to the optoelectronic properties can be achieved that range from localized modification of the conductance and generation of a photocurrent ${ }^{17,18}$ to an excitonic Mott transition ${ }^{19,20}$ to a negative photoconductivity. ${ }^{21}$ While compelling, these transient changes rely often on photoexcited populations that decay on ultrafast timescales. Alternatively, recent chemical doping schemes utilized molecular adsorbates, photoactive molecules or graphene quantum dots to inject free carriers into TMDCs when illuminated. ${ }^{22-24}$ However, in these studies, only a very limited number of carriers per molecule $(\sim 1)$ can be injected, which restricts the maximum extent of the photodoping. ${ }^{11}$

Recently, we demonstrated that the implementation of photoexcited Sn-doped $\operatorname{In}_{2} \mathrm{O}_{3}$ (ITO) nanocrystals (NCs) deposited over two-dimensional TMDCs delivers a tool for the controlled, local, and contactless carrier injection into $1 \mathrm{~L}-\mathrm{MoS}_{2}$ (i.e., p-doping). ${ }^{25}$ The key innovation is the use of ITO NCs as contactless charge injection sources that are triggered solely by light. ${ }^{25}$ In this process, after photoexcitation beyond the bandgap of ITO in the 
ultraviolet (UV) spectral range, multiple electrons are stored in one $\mathrm{NC}$ of diameter around $10 \mathrm{~nm},{ }^{26-29}$ while the corresponding holes are transferred to the $2 \mathrm{D}$ material. In this configuration, in effect, the underlying monolayer $\mathrm{MoS}_{2}$ serves as a hole collector (or electron donor). ${ }^{25}$ The charge injection area is given by the micron-sized laser spot and results in carrier density variations in the $1 \mathrm{~L}-\mathrm{MoS}_{2}$ by amounts comparable to electrostatic doping. ${ }^{1,25}$ This unique non-invasive photodoping scheme enables us to locally inject charges in an electrodeless way and with sub-micrometer precision, thus, uncovering charge relocation and propagation effects in the surrounding 2D material flake undisturbed by the influence of any solid or bulky connections - a feat that is not possible in any other 2D material doping scheme.

Within this work, we engineered a hybrid system composed of $1 \mathrm{~L}-\mathrm{MoS}_{2}$ covered in a layer of ITO NCs. We observed that in this configuration, the charge-transfer is controllable and only occurs when the NCs are excited with above-gap excitation in the UV. A single ITO NC can inject $>75$ holes to the $1 \mathrm{~L}-\mathrm{MoS}_{2}-$ a charge transfer capacity that is not attainable with any other photodoping scheme. Analysis of changes in the $1 \mathrm{~L}-\mathrm{MoS}_{2}$ photoluminescence (PL) spectra in the surrounding of the localized charge injection reveals that the local optoelectronic inhomogeneity of the $1 \mathrm{~L}-\mathrm{MoS}_{2}$ drives the doping phenomena over macroscopic distances. Spatially-dependent measurements show carrier transport and localization occurring on length scales that exceed several tens of micrometers. The resulting long-distance charge separation persists over months, making it quasipermanent. This hybrid system presents an effective method to achieve wireless, optically triggered and locally induced p-type photodoping of 2D TMDC semiconductors, and highlights the role of local inhomogeneity of 1L$\mathrm{MoS}_{2}$ on charge relocation and carrier localization. These results raise important questions as to the uniformity of carrier distribution in devices which rely on charge carrier injection where charge localization might negatively impact device performance. Thus, this work offers an in-depth understanding of devices based on 2D materials, and at the same time, highlights the opportunity of local 2D material manipulation as a step towards novel nanoscale device design. 


\section{Discussion}

\section{Photo-induced electron extraction from $\mathrm{MoS}_{2}$}

a) ITO Nanocrystals

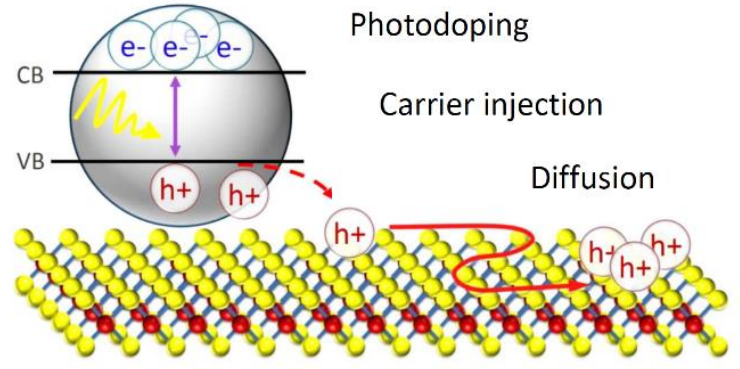

$1 \mathrm{~L}-\mathrm{MoS}_{2}$

b)

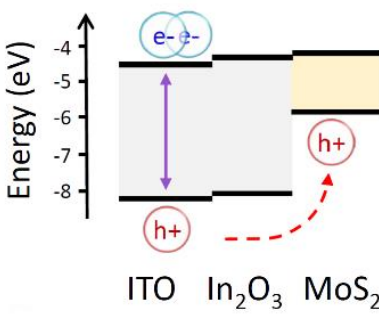

c)

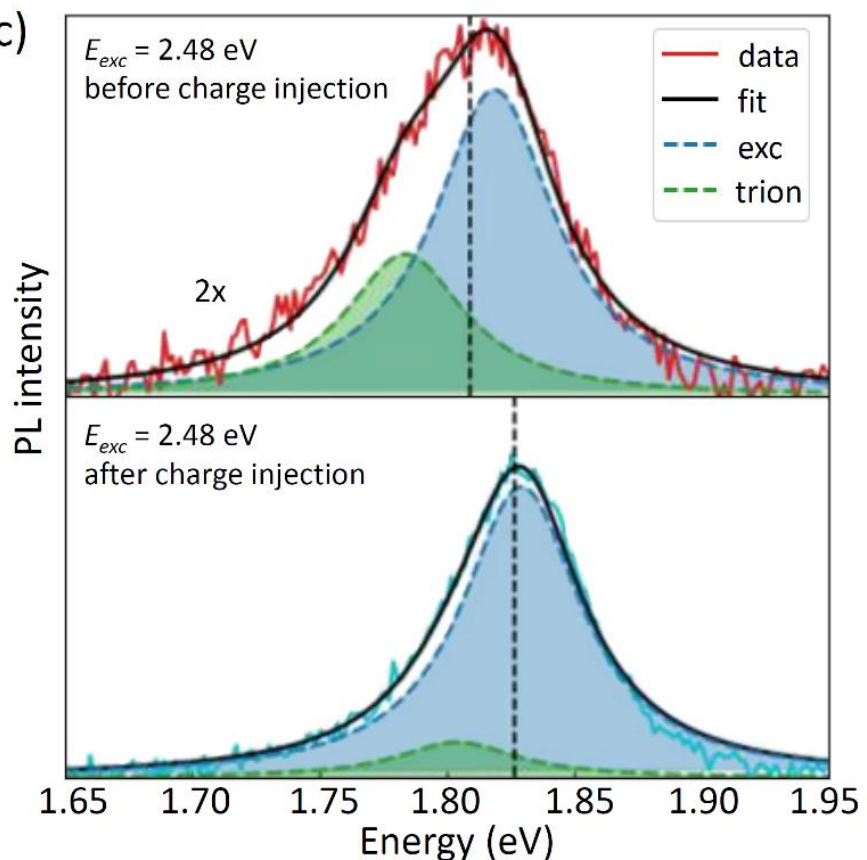

Figure 1 - Illustration of the hybrid 1L-MoS $/$ /ITO nanocrystal structure. a) After photodoping, electrons accumulate in the ITO NCs, while holes are injected into the $1 \mathrm{~L}-\mathrm{MoS}_{2}$ and diffuse away from the localized charge injection (not to scale). b) Energetic diagram illustrating the charge transfer from the ITO/ $\operatorname{In}_{2} \mathrm{O}_{3}$ core/shell NCs to the $1 \mathrm{~L}-\mathrm{MoS}_{2}$ after light absorption together with a typical SEM image (scale bar $=1 \mu \mathrm{m}$ ) of the ITO NC film covering a $1 \mathrm{~L}-\mathrm{MoS}_{2}$ flake. c) Variation of PL, measured at the same spatial position, of 1L$\mathrm{MoS}_{2}$ before (top panel) and after (bottom panel) photo-induced charge injection. The initial PL spectrum of the $1 \mathrm{~L}-\mathrm{MoS} 2$ with an excitation wavelength $\boldsymbol{E}_{\text {exc }}=2.48 \mathrm{eV}$ (no ITO excitation) is given in red. After photo-induced charge injection with UV light beyond the bandgap of the ITO NCs, the PL displays a blue shift to higher energies. The fit to the PL spectrum (black curve) is given by the sum of two Voigt line shapes, which are assigned to the exciton (blue curves) and trion (green curves) emission. The black dashed lines denote the spectral median, which captures the change in shape and energy of the emission spectrum.

Figure 1 summarizes the NC-TMDC hybrid system constructed and studied here. It is composed of ITO NCs deposited on top of CVD-grown $1 \mathrm{~L}-\mathrm{MoS}_{2}$ (Figure 1b). The hybrid structure was prepared by spin coating a concentrated solution of the NCs on the $1 \mathrm{~L}-\mathrm{MoS}_{2}$ flakes and then by depositing a thin polymer protection layer (approximately 50-100 nm). The ITO NCs are composed of a Sn-doped $\operatorname{In}_{2} \mathrm{O}_{3}$ core surrounded by a shell layer of undoped $\mathrm{In}_{2} \mathrm{O}_{3}{ }^{30-32}$ This shell passivates surface traps and inactive dopants at the NC surface and additionally aims to create a favorable heterostructure band alignment that reduces electron-hole recombination by promoting hole transfer to the surface, while localizing electrons to the core. ${ }^{25} \mathrm{NC}$ synthesis and characterization are provided in the 'Methods' section.

Figure 1c shows the PL spectrum of the $1 \mathrm{~L}-\mathrm{MoS}_{2}$ before and after exciting the hybrid system with UV excitation (see the 'Methods' section). The optical response of a $1 \mathrm{~L}-\mathrm{MoS}_{2}$ flake at a single focused laser spot with excitation energy of $2.48 \mathrm{eV}$ (below the bandgap of the NCs) was recorded prior to UV excitation (top panel of Figure 1c). After the light-driven charge injection process was triggered with UV light in the same spot for several minutes (bottom panel in Figure 1c), a blue shift and increase in intensity is observed. Comparing the two spectra, it is clear that the PL spectrum not only increases in intensity and shifts in energy, but also changes lineshape. The PL spectra of $1 \mathrm{~L}-\mathrm{MoS}_{2}$ can be deconvolved into emission bands from two states, which we assign to a low-energy trion and high-energy neutral exciton (blue and green curves Figure 1c). ${ }^{1,11,24,33}$ We observe a 
significant contribution of trion PL before photodoping, which is indicative of an initially high density of free carriers in the $1 \mathrm{~L}-\mathrm{MoS}_{2}{ }^{1,32,34}$ As-prepared $1 \mathrm{~L}-\mathrm{MoS}_{2}$ is typically reported to be $\mathrm{n}$-doped, ${ }^{1,35}$ so the trions are composed of a neutral exciton bound to an additional electron. After the charge injection process, the relative spectral weight of the trion is decreased and the exciton contribution dominates. This reduction of trions results in an overall PL intensity increase, since the quantum yield of neutral exciton emission is higher than that for trions, thus increasing the overall PL efficiency of the $1 \mathrm{~L}-\mathrm{MoS}_{2}{ }^{1,33}$ These changes in the emission spectrum, which do not occur for UV excitation of the $1 \mathrm{~L}-\mathrm{MoS}_{2}$ alone (Figure S1), indicate that the UV excitation indeed results in the removal of free electrons from the $1 \mathrm{~L}-\mathrm{MoS}_{2}$, likely through a photoinduced p-doping due to a hole transfer from the ITO NCs as sketched in Figs. 1a and $1 \mathrm{~b}$ and explained in detail in Ref. $\left[{ }^{25}\right]$.

\section{Spatial effect of photo-induced carrier injection}

Next, we consider the spatial diffusion of photo-injected holes away from the localized injection spot. To this end, we track changes in the shape of the emission spectrum using hyperspectral micro-PL mapping to generate spatially resolved images of the spectral median of the local PL spectrum. The spectral median is defined as the energy that divides the spectrum into equal amounts of high and low-energy emission, therefore capturing both peak shifts and changes in lineshape and is employed to help visualize the spatial dependence and propagation of the photodoping process (see below). In many cases, we found that spatially localized photo-induced carrier injection from ITO NCs to the $1 \mathrm{~L}-\mathrm{MoS}_{2}$ decreases the carrier density in $1 \mathrm{~L}-\mathrm{MoS}_{2}$ in regions that are several micrometers away from the photoexcited region and thus not directly illuminated.

Figure 2 provides one such example. Prior to the photodoping process, local PL spectra were recorded (i.e., mapped) over the entire extent of the flake (Figure 2a). We used $2.48 \mathrm{eV}$ excitation (which is below the bandgap of the ITO NCs) to avoid triggering the photodoping process and only excite the underlying $\mathrm{MoS}_{2}$. As observed in multiple previous studies, ${ }^{4,6,8,9,12,36,37}$ the $\mathrm{PL}$ of the $\mathrm{MoS}_{2}$ is spatially inhomogeneous. Here, we define a border between an "edge region" and an "interior region" by the contour (black line, Figures 2a, b, c) that traces the midpoint of the range of emission energies (i.e., a spectral median of $1.809 \mathrm{eV}$ in the original PL map). Before the photodoping process, the edge region exhibits a red-shifted PL emission compared to the interior region, which may be attributed to an initially higher carrier density in the edge region, but also locally increased strain and/or defect densities. ${ }^{8,12,36,38}$ Following this initial PL mapping of the hybrid $\mathrm{NC} / \mathrm{MoS}_{2}$ flake, a position in the central region (marked by the star symbol in Figure 2a) was locally illuminated with focused UV excitation $(r<$ $2.5 \mu \mathrm{m}$ ) for 5 minutes to trigger the photodoping process. Typical charging kinetics of the system have been previously investigated and the temporal response of the photo-doped hybrid has been reported in our recent work. ${ }^{25}$ Figure $2 \mathrm{~b}$ shows the spatially-dependent PL energy of the $1 \mathrm{~L}-\mathrm{MoS}_{2}$ after the localized photodoping at the center of the flake. Again, we used $2.48 \mathrm{eV}$ excitation below the bandgap of the ITO NCs to map the changes and simultaneously avoid re-excitation. Notably, the PL of the entire flake is shifted to higher energies after the photodoping (observed as a color change from yellow/red to blue/green in Figs $2 \mathrm{a}$ and b).

Figure $2 \mathrm{c}$ summarizes the PL properties as histograms of the spectral median. Before UV photodoping, the initial 2D material flake displays a broad distribution of peak positions centered around $1.81 \mathrm{eV}$ (red histogram). After localized UV exposure, the distribution narrows significantly and centers around $1.83 \mathrm{eV}$ (blue histogram). On average, the photodoping process shifts the emission energy by $17 \mathrm{meV}$, and the maximum shift reaches values up to $30 \mathrm{meV}$ (green histogram). The spatially-dependent changes in the $1 \mathrm{~L}-\mathrm{MoS}_{2}$ PL are summarized by the difference plot in Figure 2d. Although, we photoexcited only a localized spot in the center region with UV light, the most dramatic changes in the $1 \mathrm{~L}-\mathrm{MoS}_{2} \mathrm{PL}$ occur in the edge region. Strikingly, the region where the hybrid $\mathrm{NC} / \mathrm{MoS}_{2}$ system was illuminated with UV excitation at the center exhibits nearly no change in the PL spectrum (see Figure S2 of the Supporting Information for the temporal evolution of the spectra at the center of the flake), 
while the biggest changes are observed towards the edges of the flake (c.f. Figure 1c, which is a representative example of the PL variations at the edge of the flake). Furthermore, the intensity of the PL in the edge region increases significantly after localized UV exposure (Figure S2a). These results indicate that the effect of the photo-induced electron extraction from the $\mathrm{MoS}_{2}$ flake is not confined to the UV excitation spot of the laser. Importantly, we note that no such changes were observed in the $1 \mathrm{~L}-\mathrm{MoS}_{2}$ PL under UV excitation without the NCs (Figure S1).
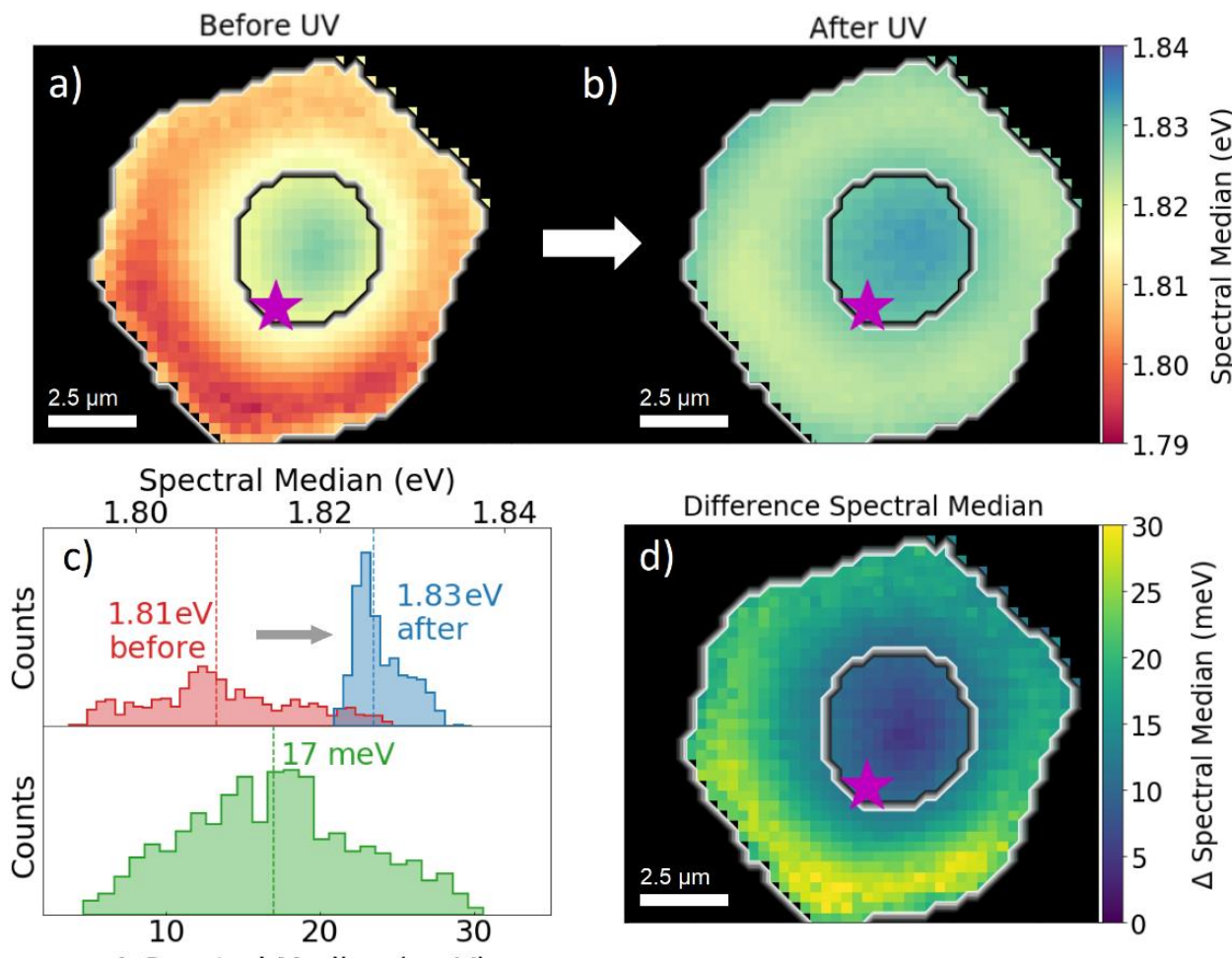

$\triangle$ Spectral Median (meV)

Figure 2 - Spatial effect of photo-induced carrier extraction in a hybrid NC/MoS $\mathbf{2}$ flake. a) Variation in the spectral median of the PL over a $1 \mathrm{~L}-\mathrm{MoS}_{2} / \mathrm{ITO} \mathrm{NC}$ hybrid flake a) before and b) after exposure to a focused UV laser spot for 5 minutes at the location marked with a star. Substantial differences in the spectral median between the edge and core region of the flake (illustrated by black contours in the plots as guide to the eye) are obvious already before photodoping. c) Upper panel: histograms of the PL peak position before (red) and after (blue) photodoping. Lower panel: Statistical summary of the difference in spectral median before and after. d) Hyperspectral map of

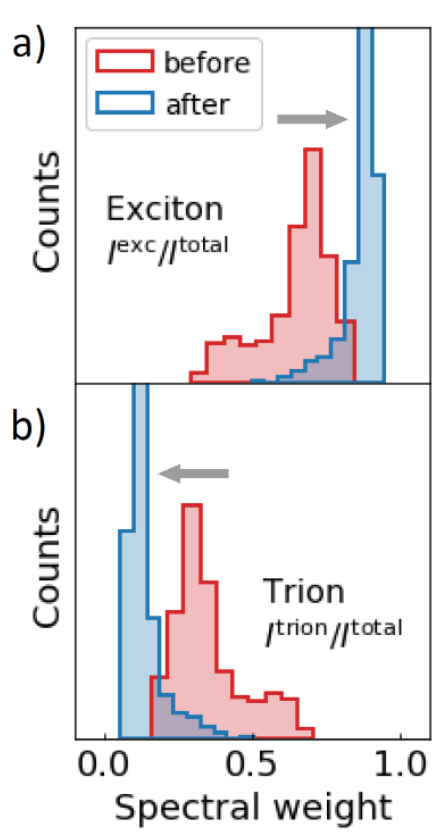

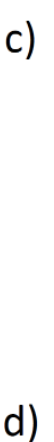

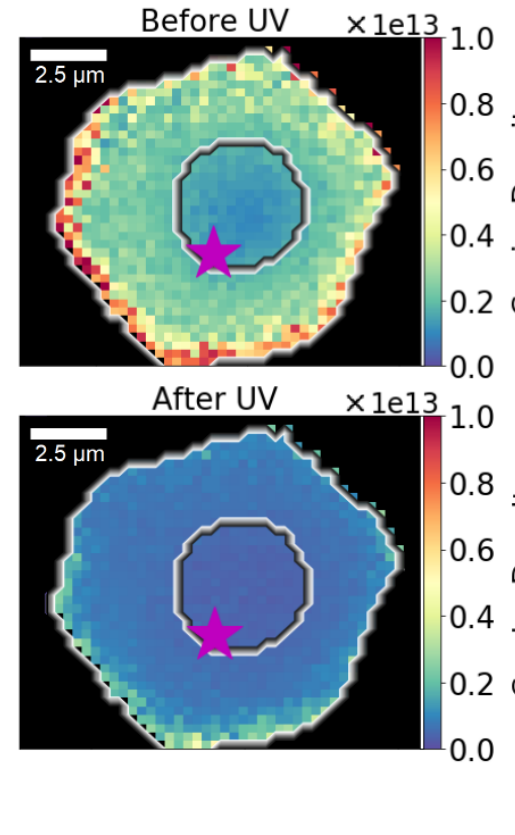

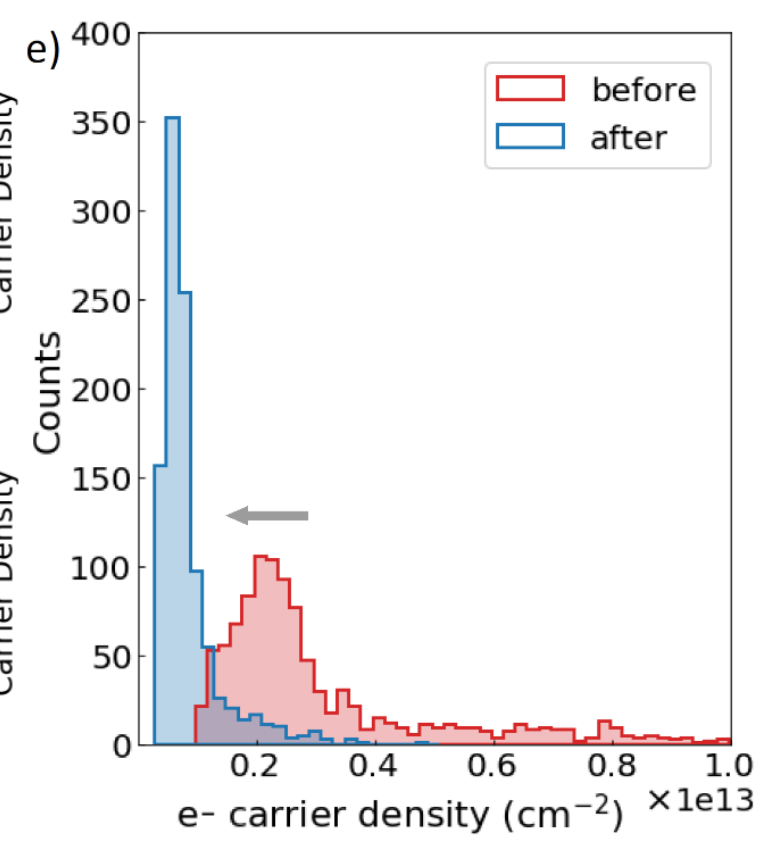


the difference of the spectral median before and after exposure to UV light. The most striking changes are observed towards the edges of the flake with peak shifts reaching $30 \mathrm{meV}$, while the core region remains fairly unchanged.

Figure 3 - Carrier density reduction in $\mathrm{MoS}_{2}$ monolayer due to UV photo-doping. a) Statistical summary of the spectral contributions of excitons and trions to the PL over the entire hybrid $\mathrm{NC} / \mathrm{MoS}_{2}$, before (in red) and after (in blue) the photo-doping. Quasiparticle contributions are calculated by fitting the PL emission with two Voigt line profiles and by separately plotting the spectral weight of the exciton (a, upper panel) and trion (b, lower panel). c) Illustration of the electron carrier density in the $1 \mathrm{~L}-\mathrm{MoS}_{2} / \mathrm{ITO}$ flake before (c) and after (d) exposing the hybrid system to UV light. The impact of the photodoping is evident in the sharp reduction of carriers in the 1L$\mathrm{MoS}_{2}$ flake, leading to a more smooth spatial distribution of the carrier density. e) Variation in the electron carrier density before (red) and after (blue) the photodoping. The initial broad distribution shifts significantly and flattens onto low carrier density values $\left(n<10^{12} \mathrm{~cm}^{-}\right.$

${ }^{2}$ ) due to the injection of photo-holes in the system.

In Figs. $3 \mathrm{a}$ and $3 \mathrm{~b}$, we report histograms of the exciton (upper panel) and trion (lower panel) contribution to the PL spectra, extracted from the deconvolution of the emission bands into trion and exciton emission for each point of the map (see Methods for details on the fitting). Initially, the trion PL contributes to the spectra with a broad distribution of its spectral weight (red histograms), representing the initial inhomogeneity of the flake. After photodoping (blue histograms) the exciton emission is enhanced at the expense of the trion emission, as a result of the photo-induced hole transfer from the ITO NCs to the $1 \mathrm{~L}-\mathrm{MoS}_{2}$. We exploited this deconvolution of room temperature PL into its exciton and trion components to estimate the carrier concentrations. This estimation allows us to extract information on the variation of the level of doping in the $1 \mathrm{~L}-\mathrm{MoS}_{2}$ from the change in the relative exciton-to-trion ratio $\left(I^{\text {exc }} / I^{\text {trion }}\right){ }^{1,11,24,33}$ By applying the mass action model, which describes the equilibrium of excitons $\left(n_{x}\right)$, trions $\left(n_{x-}\right)$ and free carriers $\left(n_{e}\right)$ in the system, we calculate the initial electron carrier density of the flake (Figure $3 \mathrm{c}$ ) and the final carrier density after the photodoping (Figure $3 \mathrm{~d}$ ). ${ }^{25}$ From the comparison between the two carrier density maps, it is evident that the photodoping of the ITO NCs in the hybrid $1 \mathrm{~L}-\mathrm{MoS}_{2} / \mathrm{ITO}$ system leads to a sharp reduction of the extra carriers in the $1 \mathrm{~L}-\mathrm{MoS}_{2}$ flake. Remarkably, the final concentration of charges in the semiconductor is more homogenous than the initial one, with photodoping leading to a smoother spatial distribution of the carrier density. Figure $3 e$ reports a histogram of the local carrier density of the system before and after the photodoping, highlighting the shift to low densities $\left(n<10^{12} \mathrm{~cm}^{-2}\right.$ ) and the associated increase in homogeneity due to the reduction of the initial broad high carrier density. We extract an average decrease in carrier density of $\sim 2.3 \times 10^{12} \mathrm{~cm}^{-2}$, which is in the range of carrier density changes achieved in electrostatically doped $\mathrm{MoS}_{2}{ }^{1,34}$, with maximum values up to more than $1 \times 10^{13} \mathrm{~cm}^{-2}$. To check the long-term stability of the photodoping and carrier diffusion effects, we measured the same hybrid flake before, immediately after and 38 days after the UV excitation (see Figure S4). We find that the photoinduced hole doping is quasi-permanent, i.e., stable on a timescale of months as already reported in our previous work. $^{25}$ Notably, we observed comparable photo-induced variations in similar systems in which we varied the position of the illumination spot on the flake (Figure S5). No significant influence on the carrier density manipulation was found from the local injection point, but it rather corresponds to the initial level of $n$-doping in the $2 \mathrm{D}$ material flake.

\section{Large spatial extent of photo-induced hole injection}

To study the spatial extent of the photo-doping in the $1 \mathrm{~L}-\mathrm{MoS}_{2} / \mathrm{ITO}$ hybrid structures, we examine the effect in a larger-area $\left(\sim 40 \times 60 \mu \mathrm{m}^{2}\right)$ polycrystalline $1 \mathrm{~L}^{-\mathrm{MoS}_{2}}$ as shown in Figure 4 . This spatially extended system is more representative of a continuous film of $1 \mathrm{~L}-\mathrm{MoS}_{2}$ and is composed of multiple $1 \mathrm{~L}-\mathrm{MoS}_{2}$ flakes that have merged together to form a network of micron-sized crystalline domains separated by grain boundaries. As above, we recorded the spatially dependent PL spectrum of the $1 \mathrm{~L}-\mathrm{MoS}_{2}$ area before and after focused UV excitation to trigger the photodoping process (purple star in Figs. 4a and b). The difference map of the entire area (Figure 4b) shows that after photodoping, the spectral median of the PL increases by up to $30 \mathrm{meV}$ over impressive distances $>40 \mu \mathrm{m}$ away from the micrometer-sized excitation spot where the photodoping was performed. Notably, the impact of local UV exposure of this hybrid NC/1L-MoS ${ }_{2}$ system is not spatially uniform and the most prevalent 
effects coincide with the grain boundaries between the individual $\mathrm{MoS}_{2}$ flakes. The correlation between the initial energy of the $1 \mathrm{~L}-\mathrm{MoS}_{2}$ PL emission and the magnitude of the photo-induced blue-shift in the spectral median is shown in Figure S6 (Supplementary Information) displaying an inverse trend with larger variations observed for regions with lower initial PL energy. To further investigate this correlation, we fitted the entire spectral map to split the PL spectra into its contribution of exciton and trion PL in analogy to what we performed above. Strikingly, the highest trion population is located close to grain boundary regions and vanishes after localized UV exposure, as illustrated by the relative increase in PL intensity along the grain boundaries (Figure S3c and d of the Supporting Information).

From the fitting of the PL spectra, we again extract the carrier density of the $1 \mathrm{~L}-\mathrm{MoS}_{2}$ for each point of the map and display in Figure S6a (Supplementary Information) the variation of carrier density before and after UV exposure. A statistical representation (normalized counts) of the carrier density changes versus distance from the excitation spot is shown in Figure 4d. At the extreme, changes in the carrier density on the order of $\sim 1 \times 10^{13} \mathrm{~cm}^{-2}$ are detected more than $40 \mu \mathrm{m}$ away from where the photodoping was performed.

a)

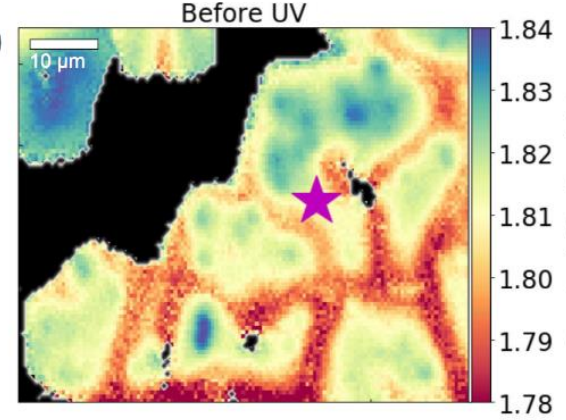

Difference Spectral Median

c)

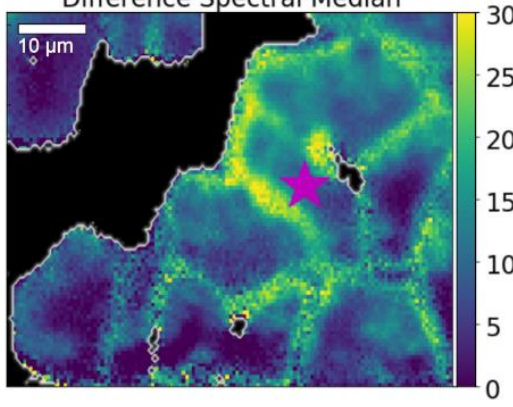

b)
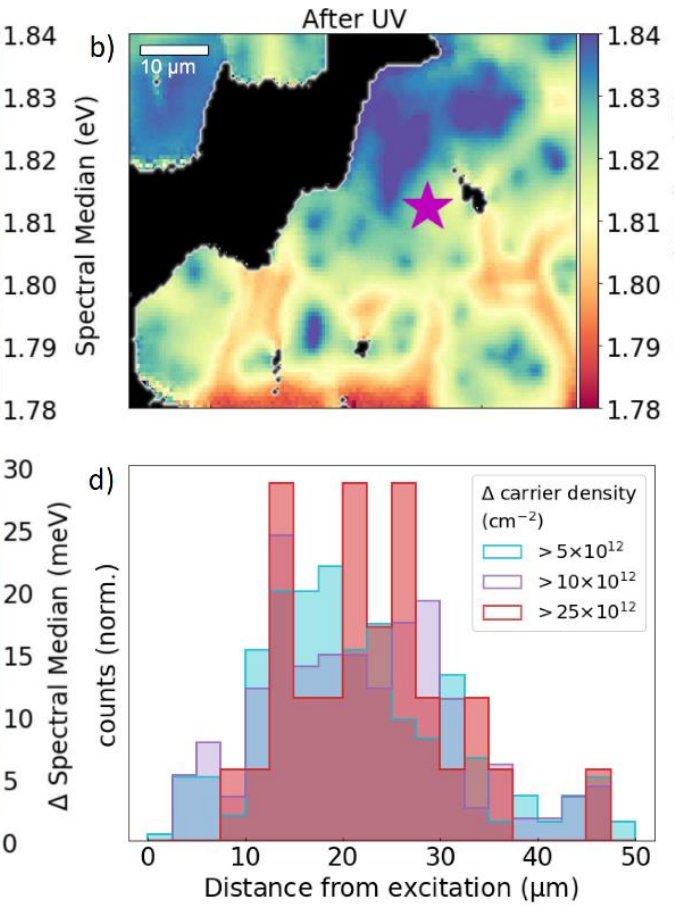

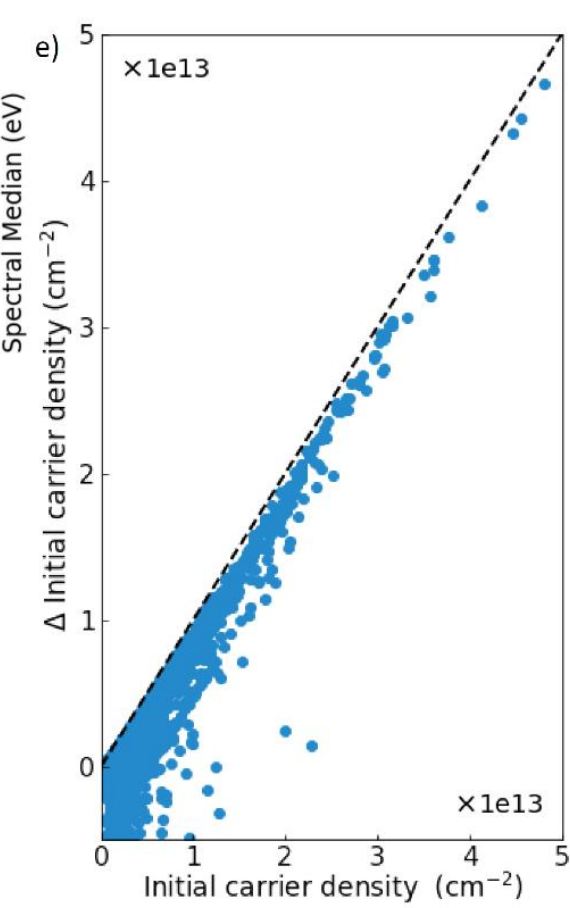

Figure 4 - Spatial extent of photo-induced electron extraction: tens of micrometers in distance and preferentially along grain boundaries. a) Spectral median of a large area of the $1 \mathrm{~L}-\mathrm{MoS}_{2} / \mathrm{ITO}$ hybrid with multiple connected flakes before (a) and after (b) the exposure to the UV laser source. Photo-doping was performed at the purple colored star in a micrometer-sized spot. Scale bar $=10 \mu \mathrm{m}$. c) Map of the photo-induced variations of the spectral median of the $1 \mathrm{~L}-\mathrm{MoS}_{2} \mathrm{PL}$, reaching changes of more than $30 \mathrm{meV}$. The impact of the photo-injected carriers into the 2D material is not spatially uniform and it is stronger along edges and grain boundaries of the flake. $\mathrm{d}$ ) Normalized statistical correlation of the magnitude of the change in the initial carrier density $(\Delta)$ with the distance from the excitation spot. We note that significant variations reach distances of more than $40 \mu \mathrm{m}$, and that the magnitude of $\Delta$ is not strongly correlated to the distance from the point where photodoping was conducted. e) Reduction in the carrier density as a function of the initial carrier density of the $1 \mathrm{~L}-\mathrm{MoS}_{2}$. The striking 1-1 linear relation between the maximum charge density variations induced and initial concentration of extra charges suggests that it is possible to completely undope the monolayer and restore a zero-doping condition in the semiconductor, regardless of the initial carrier concentration or of the distance from the excitation source. Dashed line shows the $y=x$ function, as a comparison. 
Remarkably, we do not observe a strong correlation between the change in the carrier density (as well as the spectral median) and the distance from the localized charge injection (Figure S7). This lack of correlation indicates that distance is not the limiting factor for the accumulation of carriers in specific locations of $1 \mathrm{~L}-\mathrm{MoS}_{2}$. Figure $4 \mathrm{e}$ reports the photo-induced variation in the carrier density as a function of the initial carrier density, highlighting that the maximum reduction of charges is exactly equal to the initial carrier density. Even with such a large area involved, composed of multiple flakes merged together, the striking 1-to-1 linear relation between the initial carrier concentration and the maximum variation suggests that the $1 \mathrm{~L}-\mathrm{MoS}_{2}$ tends to return to the intrinsic doping condition, regardless of the distance from the excitation source. In fact, the photo-injected holes annihilate the extra electrons in the $1 \mathrm{~L}-\mathrm{MoS}_{2}$, lowering the carrier density to intrinsic values $\left(n<2 \times 10^{12}\right)^{39-41}$ in an area of more than $2200 \mu \mathrm{m}^{2}$ (Figure S6) with a micrometer-sized excitation spot.

From these observations, we hypothesize that the driving force of the relocation of the carriers within the single layer is the initial local concentration of extra electrons leading to diffusion currents and annihilation of the locally enhanced carrier density. While this explanation consistently describes most of the experimental aspects presented, it does not provide a complete picture of the dynamics of the system. Ideally, we could expect the hybrid to reach a perfectly uniform distribution of the carrier densities (and spectral medians) after the photodoping, while the final maps still present inhomogeneities. There are several possible contributions to the deviations we observe, as the electronic landscape of single layers is extremely sensitive to local effects, including higher defect density (such as $\mathrm{S}$ vacancies) as well as inhomogeneous strain due to interactions with the underlying substrate. ${ }^{8,9,36}$ Here, Raman spectroscopic imaging (Figure S8 in the Supplementary information $)^{10,13,37}$ indicates that the edges and other regions with the largest photo-induced increase in spectral median have lower tensile strain. Strain effects on the band structure of monolayer TMDCs are welldocumented $^{9,13,14,37,38}$ showing that tensile strain relaxation increases the quasiparticle energies for electrons and holes at the K point. ${ }^{13}$ In our case, such an increase in the valence band energy would provide a driving force to re-equilibrate electrons from the edges and grain boundaries as electrons are extracted from the center of the flake due to the localized photodoping. ${ }^{10,37,38}$ However, the extent of the PL changes observed by us after photo excitation are larger than the spatial variation of the strain, suggesting that the electronic structure in monolayer TMDC flakes varies more gradually towards the center of the flakes than observed in the strain (Figure S 8). Moreover, we have to consider the possibility that the photodoping process was not complete and hence not enough photo-holes were injected into the monolayer to compensate for all the extra electrons naturally present in the material. This would explain the discrepancy observed in the maximum variation in carrier density (Figure $4 \mathrm{e})$ and could represent a limiting factor to the maximum area affected with a given power provided at the excitation spot.

Finally, we give a quantitative estimate of the photo-induced carrier injection into the $1 \mathrm{~L}-\mathrm{MoS}_{2}$ from the ITO NCs and the processes involved. As shown in Figs. 2, 3 and 4, delocalized variations in carrier density are observed over the entire flake, favoring the outer regions of the flake. Analysis of the exciton/trion spectral weight over the entire area investigated in Figure 4 by taking into account only those areas where the change in the spectral median was larger than $10 \mathrm{meV}$, we calculated the total number of transferred charges, which corresponds to 50 to 100 transferred holes per NC (see Supporting Information for more detail). We highlight here that reaching UV photo-induced charging of optimized NCs can result in up to 250 carriers per $\mathrm{NC}^{26}$, and thus that our light-induced charge injection scheme might be further optimized by overcoming intrinsic limitations by the NCs themselves. Despite having detected carrier density variations in $1 \mathrm{~L}-\mathrm{MoS}_{2}$ only up to the initial natural doping level, we anticipate that further p-type doping can be obtained by choosing optimized materials systems or through an external biasing of the $2 \mathrm{D}$ semiconductor. 


\section{Summary}

To summarize, in this work, we report a novel all-optical scheme to locally inject carriers into monolayer semiconductors in a contactless way and with micrometer precision. The advances in this work are made possible by the cooperative electronic properties of the ITO NCs and the monolayer $\mathrm{MoS}_{2}$. Quasi-permanent changes of the $\mathrm{MoS}_{2}$ carrier density (by $\sim 1 \times 10^{13} \mathrm{~cm}^{-2}$ ) are obtained through the injection of $>75$ holes per ITO NC. The effects are observed over surprisingly long distances up to $40 \mu \mathrm{m}$ away from the local (micron sized) excitation spot, covering an area of thousands of $\mu \mathrm{m}^{2}$. Carrier relocation and accumulation coincides with initially enhanced n-type doping and preferentially along edges and grain boundaries, presumably related to the presence of defects or vacancies. The striking 1-to-1 relation between the initial carrier density and its maximum variation suggests that the $1 \mathrm{~L}-\mathrm{MoS}_{2}$ tends to reach more uniform doping conditions, regardless of the distance from the excitation source. Our results show that remote light-driven doping is a powerful tool to reduce electronic inhomogeneities and enhance PL emission efficiency in $1 \mathrm{~L}-\mathrm{MoS}_{2}$ and highlights an important role of the structural environment of TMDCs. This new tool to locally inject carriers introduces possibilities to remotely manipulate the electronic structure of 2D semiconductors with micrometer precision, and to locally investigate diffusion and transport dynamics in 2D materials. Furthermore, this photo-doping process combined with local electronic structure control of TMDCs opens new functionality and application spaces for monolayer TMDC hybrids for energy conversion, exciton funneling or quantum optics. 


\section{Methods}

\section{ITO Nanocrystal synthesis}

A standard well-known synthesis was followed for the preparation of ITO/ $\mathrm{In}_{2} \mathrm{O}_{3}$ core/shell nanocrystals. ${ }^{36,37}$ The precursor solution for the doped core was prepared mixing in one vial tin(IV) acetate and indium(III) acetate with a 1:9 Sn:In ratio. Oleic acid was added in a 1:6 metal to acid ratio to yield a $10 \%$ Sn doped ITO precursor solution. The precursor solution for the undoped shell was prepared mixing indium(III) acetate with oleic acid in a 1:6 molar ratio. Both vials were left degassing at $150{ }^{\circ} \mathrm{C}$ under $\mathrm{N}_{2}$ for several hours. The ITO nanocrystal cores were synthesized by injecting the ITO precursor solution drop by drop (rate $=0.35 \mathrm{~mL} / \mathrm{min}$ ) via a syringe pump to $13.0 \mathrm{~mL}$ of oleyl alcohol at $290{ }^{\circ} \mathrm{C}$. When the synthesis of the ITO cores was completed a small aliquot was collected for analysis, showing an initial core size of $5.9 \mathrm{~nm}$. Similarly, undoped indium oleate precursor was then injected into the solution with the siring pump allowing the nanocrystals to grow to a final size of $10.1 \mathrm{~nm}$. Nanocrystals were washed with $\sim 12 \mathrm{~mL}$ ethanol and centrifugated at $7000 \mathrm{rpm}$ for $10 \mathrm{~min}$, twice.

To characterize the size and the structural features of the nanocrystals transmission electron microscopy (TEM) and small-angle X-ray scattering (SAXS) were performed. SAXS measurements were conducted at SAXSess (Anton Par, Austria), operating with a $\mathrm{Cu} \mathrm{K} \alpha$ source at $40 \mathrm{kV}$ and $50 \mathrm{~mA}$ and collecting the scattered X-ray intensities with a charge-couple device detector (Roper Scientific, Germany). TEM measurements were performed with a FEI Tecnai Spirit TEM (Hillsboro, OR) operating at $120 \mathrm{kV}$ and using a Ted Pella (Redding, CA) lacey carbon grids supported by a copper mesh.

\section{$\mathrm{MoS}_{2}$ sample preparation}

The $\mathrm{MoS}_{2}$ monolayer film was prepared via powder vaporization technique, as described in similar works. ${ }^{25,42}$ Initially, the chamber is cleaned by contaminations by pumping to base pressure (18mTorr) for 5 mins. Then, 2 mg $\mathrm{MoO}_{3}$ powder (Sigma Aldirch, 99.97\%) and $200 \mathrm{mg}$ sulfur powder (Sigma Aldrich, 99.998\%) are located in two hot zones in the horizontal furnace (2' tube diameter). The carrier gas implemented for the growth is $100 \mathrm{sccm}$ Ar. During the nucleation phase the $\mathrm{MoO}_{3}$ and the substrate are heated up to $550{ }^{\circ} \mathrm{C}$ for 2 mins, the temperature is then decreased to $130{ }^{\circ} \mathrm{C}$ for the $\mathrm{S}$ powder when the nucleation step is finished. Finally, the temperature in the chamber is set to $725^{\circ} \mathrm{C}$ for 15 mins, while keeping the powder at $130{ }^{\circ} \mathrm{C}$.

\section{Hybrid sample formation}

The hybrid was prepared by spin-coating $20 \mu \mathrm{L}$ of the $10 \mathrm{mg} / \mathrm{mL}$ nanocrystal solution at 2000 rounds per minute (rpm) for 45 seconds over the $\mathrm{MoS}_{2}$ sample. To isolate the system a thin layer of polymethyl methacrylate was spin-coated on top of the hybrid by depositing $50 \mu \mathrm{L}$ of a $5 \mathrm{mg} / \mathrm{mL}$ stock solution for $45 \mathrm{sec}$ and $2000 \mathrm{rpm}$.

\section{Optical measurements}

To collect hyperspectral maps of the PL emission we used a scanning confocal microscope equipped with a $50 \times 0.6$ NA objective and coupled to a $500 \mathrm{~nm}$ pulsed laser excitation source $(2.48 \mathrm{eV} ; 80 \mathrm{MHz}$ repetition rate) The emission was collected from the sample through the same objective, filtering the (green or UV) light reflected from the laser with a $600 \mathrm{~nm}$ long-pass filter combined with a $532 \mathrm{~nm}$ long-pass dichroic mirror. The signal was then dispersed by a spectrometer (Princeton Instruments) and collected by a cooled charge coupled device (CCD) camera (Andor). Hyperspectral imaging was performed by collecting PL spectra in each spatial point of the map, raster-scanning the sample with high precision piezo stages (Mad City Labs) and collecting the data with a custom-made microscopy software. ${ }^{41}$ For all measurements, the excitation density was kept low enough to stay in the linear regime. Contactless photodoping was performed by focusing a $350 \mathrm{~nm}(3.54 \mathrm{eV})$ pulsed laser onto a fixed spot of the sample for few minutes. Raman measurements were conducted with a Raman microscope system (NTMDT), employing a CW laser excitation at $532 \mathrm{~nm}(2.33 \mathrm{eV})$ - with a power of 
$500 \mu \mathrm{W}$ - focused with a $100 \times 0.6$ NA objective. The Raman emission, collected with the same objective, was then detected and analyzed with the Raman spectroscopy system.

\section{Fit of the-photoluminescence spectra:}

To quantitative analyze the PL spectrum of the monolayer $\mathrm{MoS}_{2}$, each PL spectrum at a given time was fit with Voigt functions, representing the convolution of a Lorentzian and a Gaussian:

$$
\mathcal{V}(E, \sigma, \gamma)=\frac{\operatorname{Re}\left[w\left(\frac{E+i \gamma}{\sigma \sqrt{2}}\right)\right]}{\sigma \sqrt{2 \pi}}
$$

Where $w$ is the Faddeeva function, $\gamma$ is the half-width at half-maximum (HWHM) of the Lorentzian and $\sigma$ is the standard deviation of the Gaussian. The fit was performed considering the sum of two oscillators, representing the exciton and the trion emission:

$$
\begin{gathered}
I(E)=I_{X} \cdot \mathcal{V}\left(E-E_{X}, \sigma_{X}, \gamma_{X}\right)+I_{T} \cdot \mathcal{V}\left(E-E_{T}, \sigma_{T}, \gamma_{T}\right) . \\
I(E)=I_{X} \cdot \frac{\operatorname{Re}\left[w\left(\frac{E-E_{X}+i \gamma_{X}}{\sigma_{X} \sqrt{2}}\right)\right]}{\sigma_{X} \sqrt{2 \pi}}+I_{T} \cdot \frac{\operatorname{Re}\left[w\left(\frac{E-E_{T}+i \gamma_{T}}{\sigma_{T} \sqrt{2}}\right)\right]}{\sigma_{T} \sqrt{2 \pi}} .
\end{gathered}
$$

-Here, $\gamma_{X}$ and $\gamma_{T}$ are the half-width at half-maximum of the Lorentzian profiles while $\sigma_{X}$ and $\sigma_{T}$ are the standard deviation of the Gaussian contributes to the exciton and trion oscillators, respectively. $I_{X}$ and $I_{T}$ are prefactors that scale the overall intensity of the exciton and trion oscillators, respectively. The energetic position of the exciton oscillator, $E_{X}$, is related to the center position of the trion oscillator, $E_{T}$ by the binding energy of the trion state, $E_{b}$ :

$$
E_{T}=E_{X}-E_{b}
$$

|-In the fitting procedure, the initial undoped spectrum was fitted to extract above fitting parameters using the restraints summarized in Table $\mathrm{S} 1$.

Table S1 - Summary of fitting restraints.

\begin{tabular}{|c|c|c|c|}
\hline Parameter & Min & Max & Units \\
\hline$I_{X}$ & 0.5 & 30 & CCD Counts \\
$E_{X}$ & 1.67 & 1.88 & $\mathrm{eV}$ \\
$\gamma_{X}$ & 0 & 0.05 & $\mathrm{eV}$ \\
$\sigma_{X}$ & 0 & 0.05 & $\mathrm{eV}$ \\
$I_{T}$ & 0.5 & 30 & $\mathrm{CCD} \mathrm{Counts}$ \\
$E_{b}$ & 0.00 & 0.50 & $\mathrm{eV}$ \\
$\gamma_{T}$ & 0 & 0.05 & $\mathrm{eV}$ \\
$\sigma_{T}$ & 0 & 0.05 & $\mathrm{eV}$ \\
\hline
\end{tabular}


Fit of the spectral variation of the PL over the entire map:

The spectral fit over the flake was performed at each pixel in a similar way as described above. For the comparison before and after UV exposure we used the parameters extracted from the map before exposure at each pixel as the starting point for the fit of the same pixel after UV exposure, keeping the extracted width of the Lorentz and Gaussian peaks for the Voigt profiles associated to exciton and trion constant.

\section{Mass action model}

A model can be applied to the changes in the exciton-to-trion ratio to gain a deeper, quantitative insight into the photo-induced changes of the carrier density in the $\mathrm{MoS}_{2}$. For low excitation densities (i.e., when the density of excitons is much smaller than the density of pre-existing carriers), the thermal equilibrium of the free carrier, exciton, and trion populations is given by the Boltzmann distribution. ${ }^{42,43}$ The mass action model describing the equilibrium of excitons $\left(n_{x}\right)$, trions $\left(n_{x-}\right)$ and free carriers $\left(n_{e}\right) n_{x}+n_{e} \leftrightarrow n_{x-}$ in the system, was used to describe the variation of the carrier density in quantum well structures ${ }^{44,45}$ and adapted to layered 2D materials, such as $\mathrm{MoS}_{2}$ or $\mathrm{MoSe}_{2} \cdot{ }^{11,24,33}$ Here, we have similarly adapted this model to represent the temporal evolution of the exciton-totrion ratio, and from that, to extract the variation of the carrier density $n_{e}$ upon photodoping (Error! Reference source not found.c):

$$
n_{e}(t)=\frac{n_{\chi^{-}}(t)}{n_{\chi}(t)} \cdot \frac{4 M_{X_{0}} m_{e}}{\pi \hbar^{2} M_{\chi^{-}}} \cdot k_{B} T \cdot e^{\left(-\frac{E_{B}^{x^{-}}}{k_{B} T}\right)},
$$

where $m_{e}$ is the effective mass of the electron in $\mathrm{MoS}_{2}, M_{X_{0}}$ and $M_{x^{-}}$are the effective masses of the exciton and trion respectively, $E_{B}^{x^{-}}$is the intrinsic trion binding energy (from Ref. ${ }^{1}$ ), and $n_{e}, n_{x}$ and $n_{x-}$ denote carrier, neutral exciton and trion densities, respectively. Over the extent of the photodoping process, the carrier density decreases by $\sim 6 \times 10^{12} \mathrm{~cm}^{-2}$, which is in the range of carrier density changes achieved in electrostatically doped $\operatorname{MoS}_{2}{ }^{1,34}$ Furthermore, the final carrier density reached is approximately $1.5 \times 10^{12} \mathrm{~cm}^{-2}$, which again, in comparison to previous work on electrostatically doped $\mathrm{MoS}_{2}{ }^{1}$, agrees well with the carrier-density anticipated to yield a relative trion intensity of 0.2 with an energetic splitting between the exciton and trion of $28.5 \mathrm{meV}$.

\section{Fit of the Raman peaks:}

The Raman peaks were fitted by implementing one Lorentzian function per peak.

\section{Quantitative analysis of the average charge transferred per nanocrystal:}

To calculate the average number of photo-carriers injected per nanocrystal we normalized the total number of carriers transferred $q^{\text {tot }}$, extracted from the variations in carrier density $n_{e}$, with the number of nanocrystals exposed to UV light $N^{\text {excited }}$ We can then express the average number of carriers transferred per nanocrystal as:

$$
{ }_{c}^{\mathrm{NC}}=\frac{q^{\text {tot }}}{N^{\text {excited }}}=\frac{q^{\text {tot }}}{\pi\left(d^{\text {spot }} / 2\right)^{2} \cdot \rho^{\mathrm{NC}}}
$$

where $\rho_{\mathrm{NC}}$ is the density of the thin layer of ITO nanocrystals in the hybrid and $d^{\text {spot }}=5 \mu \mathrm{m}$ is the diameter of the area illuminated by the UV laser spot (approximated with a circle). From SEM images of the hybrid system we estimated the average concentration of nanocrystals $\rho^{\mathrm{NC}}$ in different areas of the sample. Since the density of the thin layer is not uniform we considered three different values of $\rho^{\mathrm{NC}}$ (an average value, a low value and a high value associated to a continuous packaging of nanocrystals) in order to take in account uncertainties in the assumptions and to set lower and upper boundaries to the final estimate. 
We obtained an average value of $c^{\mathrm{NC}}=75$ photo-carriers transferred per nanocrystal for the photodoped area reported in Figure 4. Within the same analysis we estimated a upper value of $c^{\mathrm{NC}}=100$ and a minimum value of $c^{\mathrm{NC}}=50$ carriers injected per nanocrystal, depending on the density of the packaging in the nanocrystal layer. In our previous work we estimated an average value of only $\sim 5$ holes per nanocrystal by considering the photoinduced variations in the area of the sample directly probed with the laser spot, ${ }^{25}$ without taking in account diffusion effects. This is another evidence that carrier diffusion plays a significant role in the dynamics of the system as the majority of the injected carriers moves away from the excitation spot.

\section{Acknowledgements}

This project has received funding from the European Union's Horizon 2020 research and innovation programme under the Marie Skłodowska-Curie Grant Agreement No. [705444] (MOPTOPus) as well as Grant Agreement No. [734690] (SONAR), and from the European Research Council Grant Agreement No. [850875] (LightDYNAMO). Work at the Molecular Foundry was supported by the Office of Science, Office of Basic Energy Sciences, of the U.S. Department of Energy under Contract No. DE-AC02-05CH11231. 


\section{References}

1. Mak, K. F. et al. Tightly bound trions in monolayer $\mathrm{MoS}_{2}$. Nat. Mater. 12, 207-211 (2013).

2. Radisavljevic, B., Radenovic, A., Brivio, J., Giacometti, V. \& Kis, A. Single-layer $\mathrm{MoS}_{2}$ transistors. Nat. Nanotechnol. 6, 147-150 (2011).

3. Basov, D. N., Averitt, R. D. \& Hsieh, D. Towards properties on demand in quantum materials. Nat. Mater. 16, 1077-1088 (2017).

4. Borys, N. J. et al. Anomalous Above-Gap Photoexcitations and Optical Signatures of Localized Charge Puddles in Monolayer Molybdenum Disulfide. ACS Nano 11, 2115-2123 (2017).

5. Amani, M. et al. Near-unity photoluminescence quantum yield in MoS2. Science 350, 1065-1068 (2015).

6. Kastl, C. et al. Multimodal spectromicroscopy of monolayer WS 2 enabled by ultra-clean van der Waals epitaxy. 2D Mater. 5, 045010 (2018).

7. Mitterreiter, E. et al. Atomistic Positioning of Defects in Helium Ion Treated Single-Layer MoS2. Nano Lett. (2020) doi:10.1021/acs.nanolett.0c01222.

8. Carozo, V. et al. Optical identification of sulfur vacancies: Bound excitons at the edges of monolayer tungsten disulfide. Sci. Adv. 3, e1602813 (2017).

9. Liu, Z. et al. Strain and structure heterogeneity in $\mathrm{MoS}_{2}$ atomic layers grown by chemical vapour deposition. Nat. Commun. 5, 5246 (2014).

10. Castellanos-Gomez, A. et al. Local Strain Engineering in Atomically Thin MoS2. Nano Lett. 13, 5361-5366 (2013).

11. Mouri, S., Miyauchi, Y. \& Matsuda, K. Tunable Photoluminescence of Monolayer MoS2 via Chemical Doping. Nano Lett. 13, 5944-5948 (2013).

12. Bao, W. et al. Visualizing nanoscale excitonic relaxation properties of disordered edges and grain boundaries in monolayer molybdenum disulfide. Nat. Commun. 6, 7993 (2015).

13. Feng, J., Qian, X., Huang, C.-W. \& Li, J. Strain-engineered artificial atom as a broad-spectrum solar energy funnel. Nat. Photonics 6, 866-872 (2012).

14. Zande, A. van der \& Hone, J. Optical materials: Inspired by strain. Nat. Photonics 6, 804-806 (2012).

15. High, A. A. et al. Condensation of Excitons in a Trap. Nano Lett. 12, 2605-2609 (2012). 
16. Gong, C. et al. Metal Contacts on Physical Vapor Deposited Monolayer MoS2. ACS Nano 7, 11350-11357 (2013).

17. Fang, H. \& Hu, W. Photogating in Low Dimensional Photodetectors. Adv. Sci. 4, 1700323 (2017).

18. Buscema, M. et al. Photocurrent generation with two-dimensional van der Waals semiconductors. Chem. Soc. Rev. 44, 3691-3718 (2015).

19. Chernikov, A., Ruppert, C., Hill, H. M., Rigosi, A. F. \& Heinz, T. F. Population inversion and giant bandgap renormalization in atomically thin $\mathrm{WS}_{2}$ layers. Nat. Photonics 9, 466-470 (2015).

20. Wang, J. et al. Optical generation of high carrier densities in 2D semiconductor heterobilayers. Sci. Adv. 5, eaax0145 (2019).

21. Lui, C. H. et al. Trion-Induced Negative Photoconductivity in Monolayer MoS2. Phys. Rev. Lett. 113, 166801 (2014).

22. Li, J. et al. Tuning the optical emission of MoS2 nanosheets using proximal photoswitchable azobenzene molecules. Appl. Phys. Lett. 105, 241116 (2014).

23. Margapoti, E. et al. A 2D Semiconductor-Self-Assembled Monolayer Photoswitchable Diode. Adv. Mater. 27, 1426-1431 (2015).

24. Li, Z. et al. Graphene Quantum Dots Doping of MoS2 Monolayers. Adv. Mater. 27, 5235-5240 (2015).

25. Kriegel, I. et al. Light-Driven Permanent Charge Separation across a Hybrid Zero-Dimensional/TwoDimensional Interface. J. Phys. Chem. C 124, 8000-8007 (2020).

26. Brozek, C. K. et al. Soluble Supercapacitors: Large and Reversible Charge Storage in Colloidal Iron-Doped ZnO Nanocrystals. Nano Lett. 18, 3297-3302 (2018).

27. Brozek, C. K., Hartstein, K. H. \& Gamelin, D. R. Potentiometric Titrations for Measuring the Capacitance of Colloidal Photodoped ZnO Nanocrystals. J. Am. Chem. Soc. 138, 10605-10610 (2016).

28. Kriegel, I. et al. Ultrafast Photodoping and Plasmon Dynamics in Fluorine-Indium Codoped Cadmium Oxide Nanocrystals for All-Optical Signal Manipulation at Optical Communication Wavelengths. J. Phys. Chem. Lett. 7, 3873-3881 (2016). 
29. Kriegel, I., Scotognella, F. \& Manna, L. Plasmonic doped semiconductor nanocrystals: Properties, fabrication, applications and perspectives. Phys. Rep. 674, 1-52 (2017).

30. Crockett, B. M., Jansons, A. W., Koskela, K. M., Johnson, D. W. \& Hutchison, J. E. Radial Dopant Placement for Tuning Plasmonic Properties in Metal Oxide Nanocrystals. ACS Nano 11, 7719-7728 (2017).

31. Jansons, A. W. \& Hutchison, J. E. Continuous Growth of Metal Oxide Nanocrystals: Enhanced Control of Nanocrystal Size and Radial Dopant Distribution. ACS Nano 10, 6942-6951 (2016).

32. Jansons, A. W., Plummer, L. K. \& Hutchison, J. E. Living Nanocrystals. Chem. Mater. 29, 5415-5425 (2017).

33. Ross, J. S. et al. Electrical control of neutral and charged excitons in a monolayer semiconductor. Nat. Commun. 4, 1474 (2013).

34. Chernikov, A. et al. Electrical Tuning of Exciton Binding Energies in Monolayer WS2. Phys. Rev. Lett. 115, $126802(2015)$.

35. Yao, K. et al. Optically Discriminating Carrier-Induced Quasiparticle Band Gap and Exciton Energy Renormalization in Monolayer MoS2. Phys. Rev. Lett. 119, 087401 (2017).

36. Kastl, C. et al. The important role of water in growth of monolayer transition metal dichalcogenides. $2 D$ Mater. 4, 021024 (2017).

37. Zande, A. M. van der et al. Grains and grain boundaries in highly crystalline monolayer molybdenum disulphide. Nat. Mater. 12, 554-561 (2013).

38. Kastl, C. et al. Effects of Defects on Band Structure and Excitons in WS2 Revealed by Nanoscale Photoemission Spectroscopy. ACS Nano (2019) doi:10.1021/acsnano.8b06574.

39. Jariwala, D. et al. Band-like transport in high mobility unencapsulated single-layer $\mathrm{MoS}_{2}$ transistors. Appl. Phys. Lett. 102, 173107 (2013).

40. Smithe, K. K. H., English, C. D., Suryavanshi, S. V. \& Pop, E. Intrinsic electrical transport and performance projections of synthetic monolayer $\operatorname{MoS}_{2}$ devices. 2D Mater. 4, 011009 (2016).

41. Kaasbjerg, K., Thygesen, K. S. \& Jacobsen, K. W. Phonon-limited mobility in n -type single-layer MoS 2 from first principles. Phys. Rev. B 85, 115317 (2012). 
42. Zhang, K. et al. Deconvoluting the Photonic and Electronic Response of 2D Materials: The Case of MoS2. Sci. Rep. 7, 16938 (2017).

43. Durham, D. B., Ogletree, D. F. \& Barnard, E. S. Scanning Auger spectromicroscopy using the ScopeFoundry software platform. Surf. Interface Anal. 50, 1174-1179 (2018).

44. Kossacki, P. Optical studies of charged excitons in II-VI semiconductor quantum wells. J. Phys. Condens. Matter 15, R471 (2003).

45. Siviniant, J., Scalbert, D., Kavokin, A. V., Coquillat, D. \& Lascaray, J.-P. Chemical equilibrium between excitons, electrons, and negatively charged excitons in semiconductor quantum wells. Phys. Rev. B 59, 1602-1604 (1999). 


\section{Supporting Information}

a)

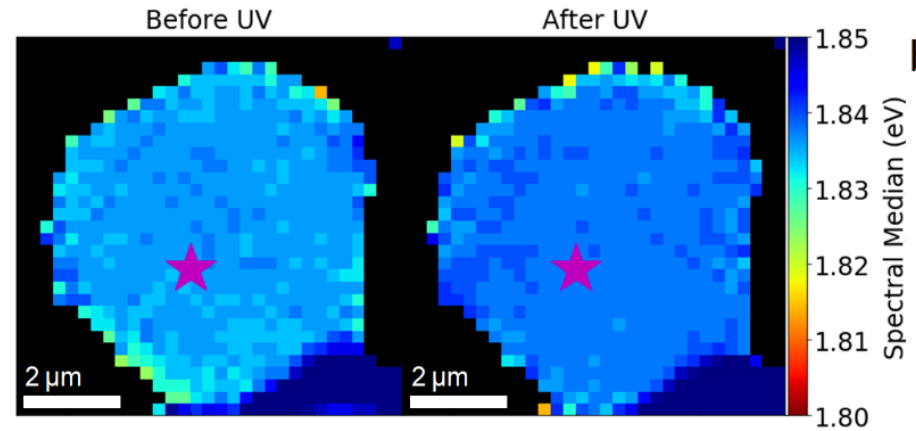

b)

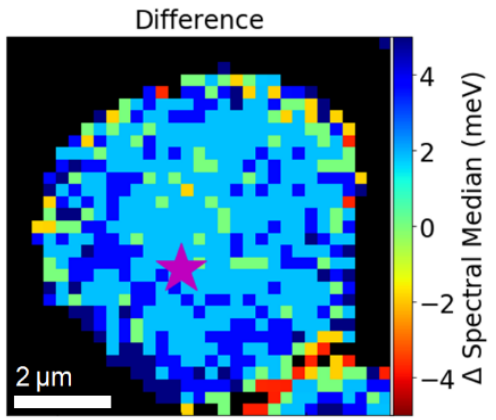

Figure S 1 Photodoping of a reference sample without ITO nanocrystals. Plot of the spectral median of the PL spectrum scanned over a single $\mathrm{MoS}_{2}$ flake without ITO nanocrystals before and after exposure to UV light. No significant variations are observed as demonstrated by the difference map. The pink star illustrates the location at which the UV (350 nm) laser was focused (with a radius of circa $2.5 \mu \mathrm{m})$.

a)

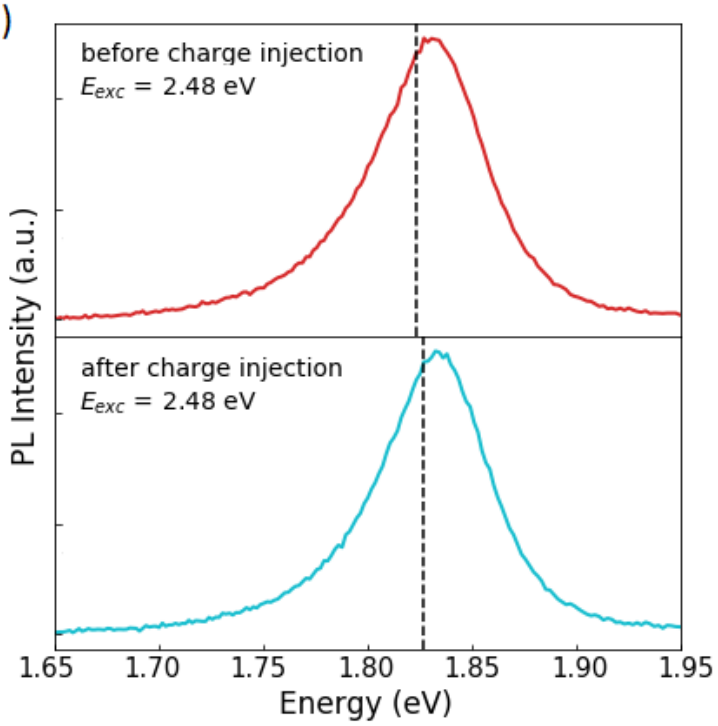

b)

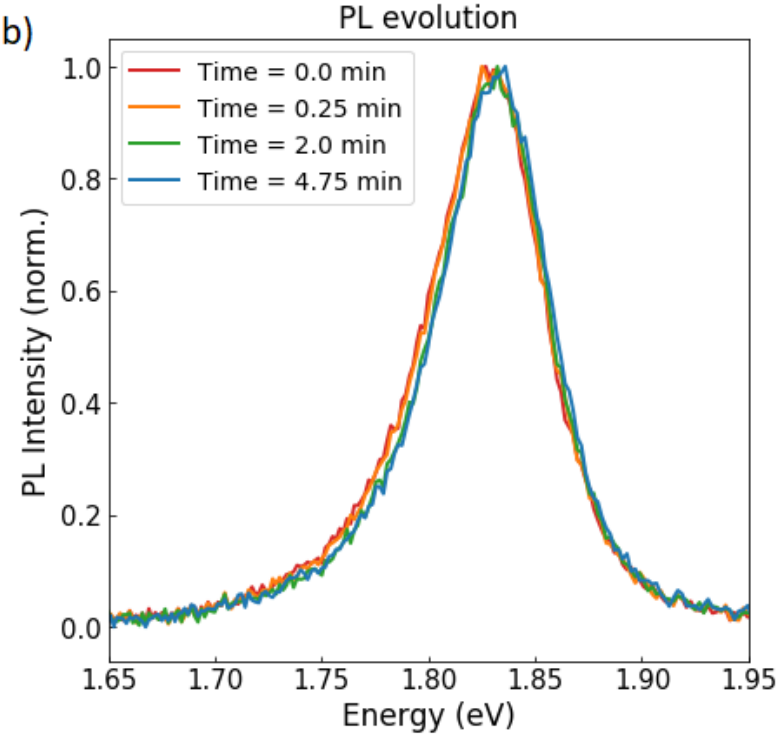

Figure S 2 Temporal evolution of the PL profile at the excitation spot. a) Emission before (upper panel) and after (lower panel) the photodoping of the flake investigated in Figure 2 collected directly at the UV excitation spot. b) Temporal evolution of the PL emission over a time scale of 5 minutes of UV exposure showing representative spectra. Strikingly, barely any changes are observed in the PL, indicating that most of the photo-injected charges diffuse from the excitation spot to farther regions of the hybrid system leaving almost unaffected the area directly illuminated with the laser. 
a)

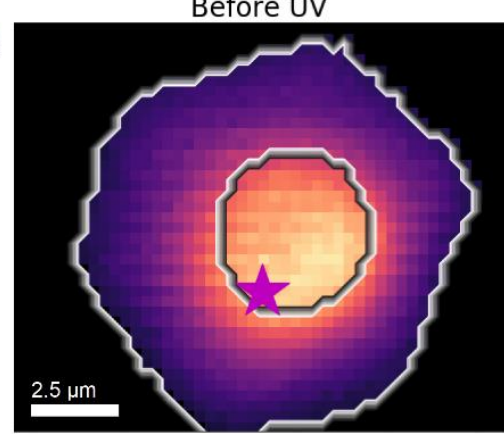

c)

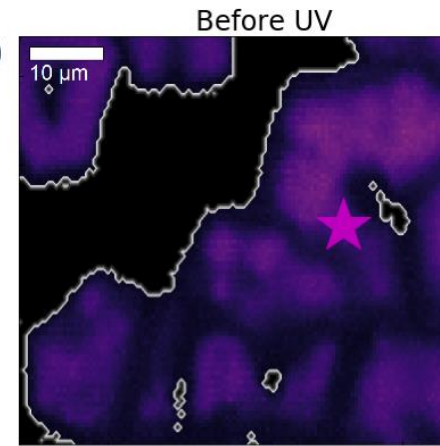

After UV

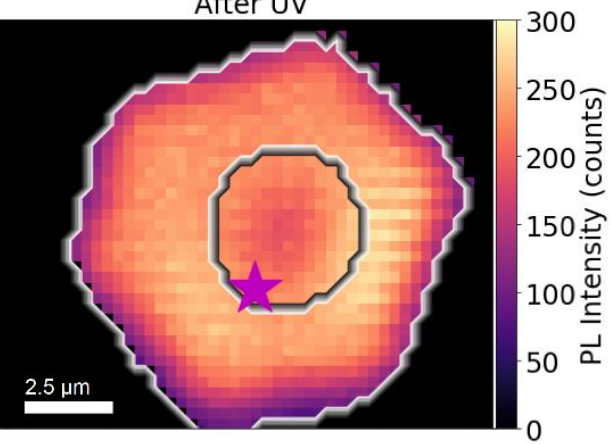

After UV

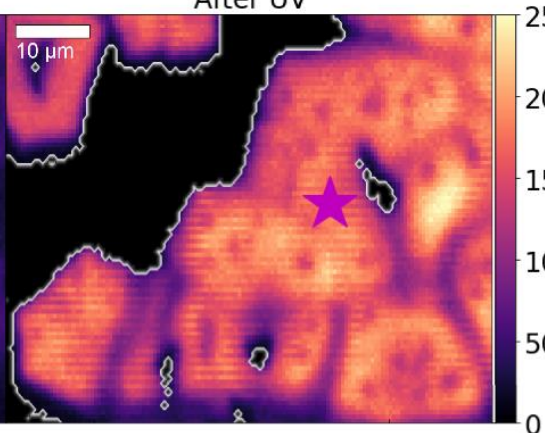

b)

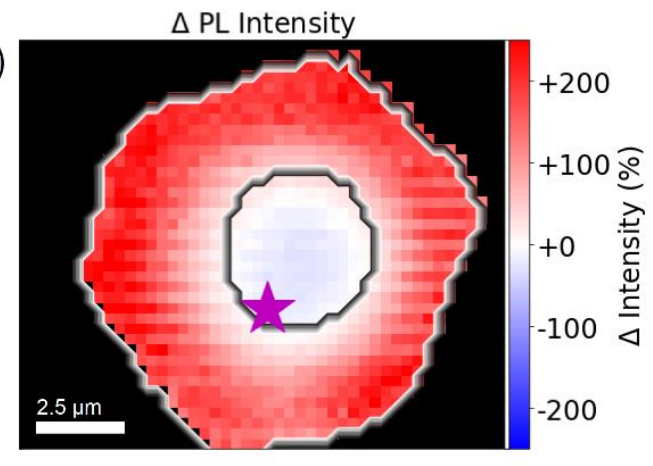

d)

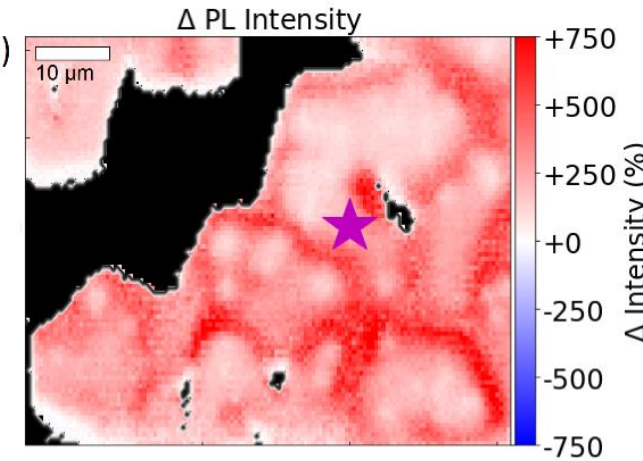

Figure S 3 Photo-induced variations in the Photoluminescence intensity. a) PL intensity before and after the photodoping of the Flake reported in Figure 2 and b) illustration of intensity increase (in percentage) normalized with respect to the initial intensity. $\Delta$ Intensity (\%) $=100 *\left(I^{\text {after }}-I^{\text {before }}\right) / I^{\text {before }}$. c) PL intensity before and after the photodoping of the area reported in Figure 4 and d) illustration of the normalized intensity increase $\Delta$ Intensity (\%). While the overall PL intensity tend to increase almost everywhere in term of absolute counts, we notice that the biggest variations in intensity percentage are localized in the areas that initially present a high concentration of trions. This is consistent with what is expected as the PL emission generated from the recombination of excitons is brighter than the same trion-based process, and it is another indicator that the formation of trions is hindered and the formation of excitons is enhanced increasing the overall photoluminescence efficiency of the $1 \mathrm{~L}-\mathrm{MoS}_{2}$.

a)

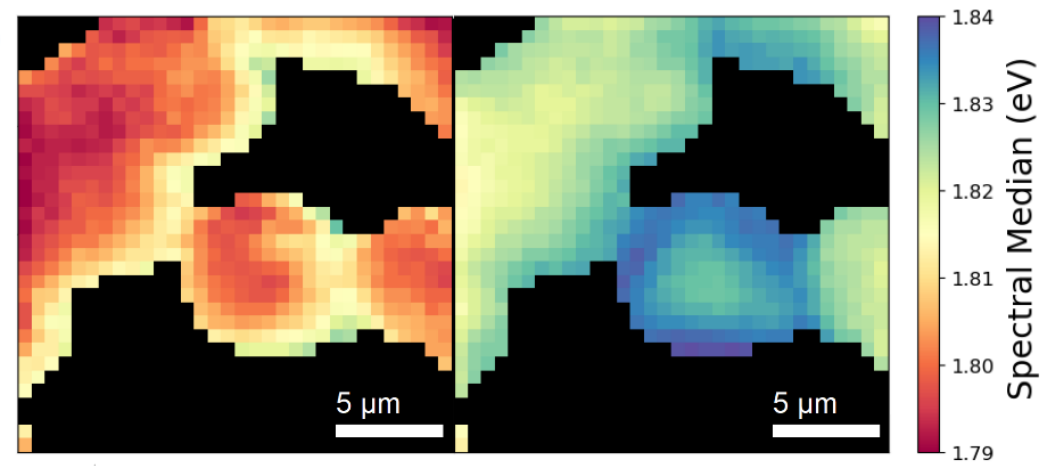

b)

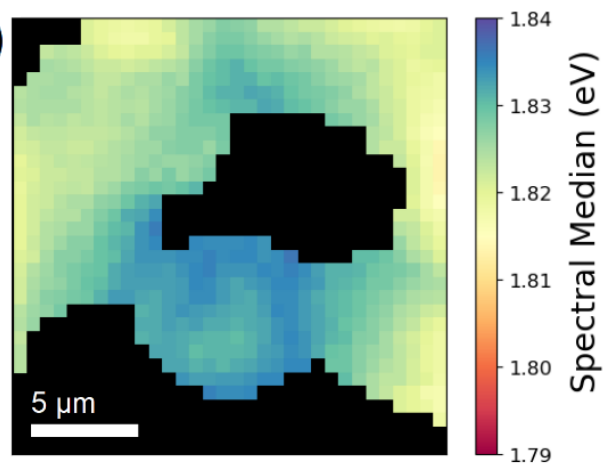

Figure S 4 Long-term stability of the photodoping effects. a) Spectral median map of a portion of the MoS2/ITO hybrid before (left) and after (right) the photodoping. b) Spectral map of the same area of the system collected 38 days later. The photo-induced effects of few minutes of UV exposure are still clearly visible in the hybrid, proving that the transfer of photo-holes is permanent and stable on a timescale of months. 
a)
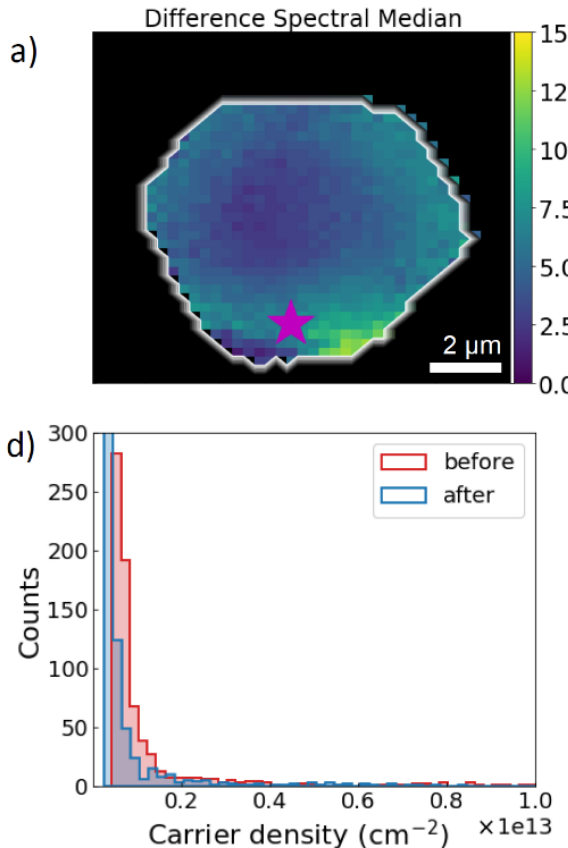

b)
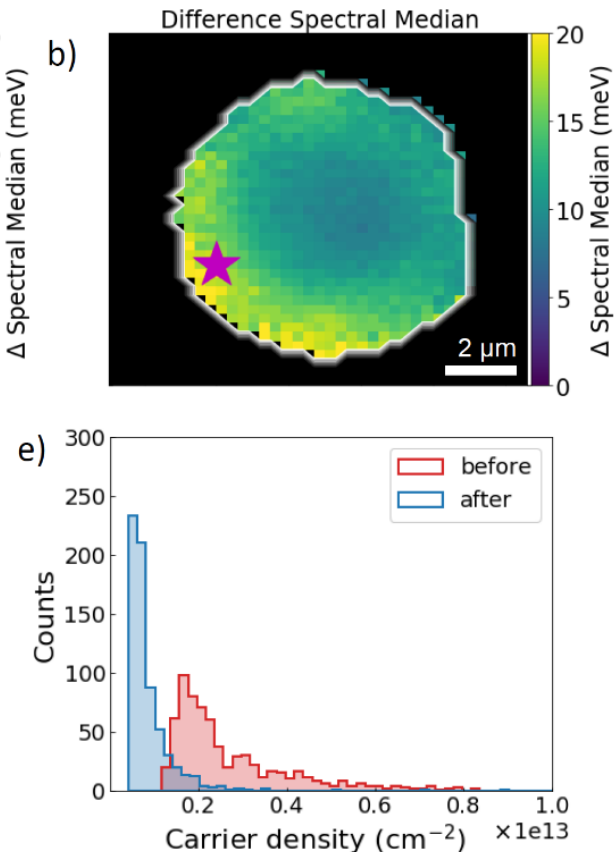

c)

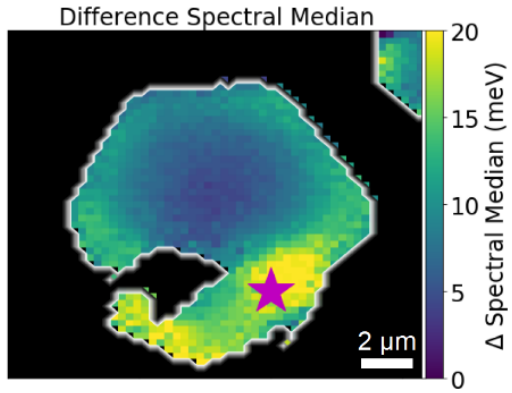

f)

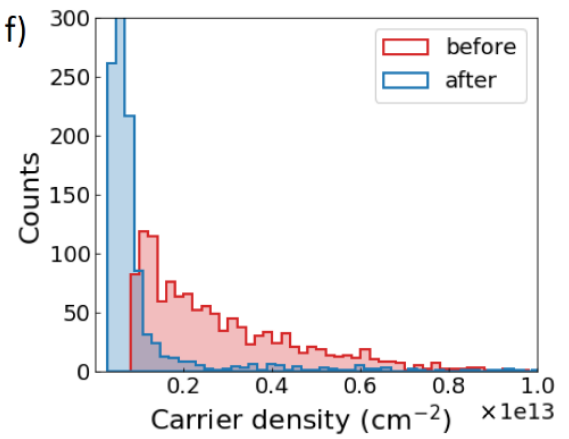

Figure S 5 Photodoping effects in various flakes with different excitation spots. Variation of the spectral median of the PL due to the photodoping for three different flakes (a-c). The laser position is indicated by a purple star for each flake. d) Statistical summary of the carrier density distribution in the same three flakes (d-e) before (red) and after (blue) the injection of photo-charges. Photodoping effects depend on the initial carrier density distribution and occurs irrespectively from the injection location.

a)
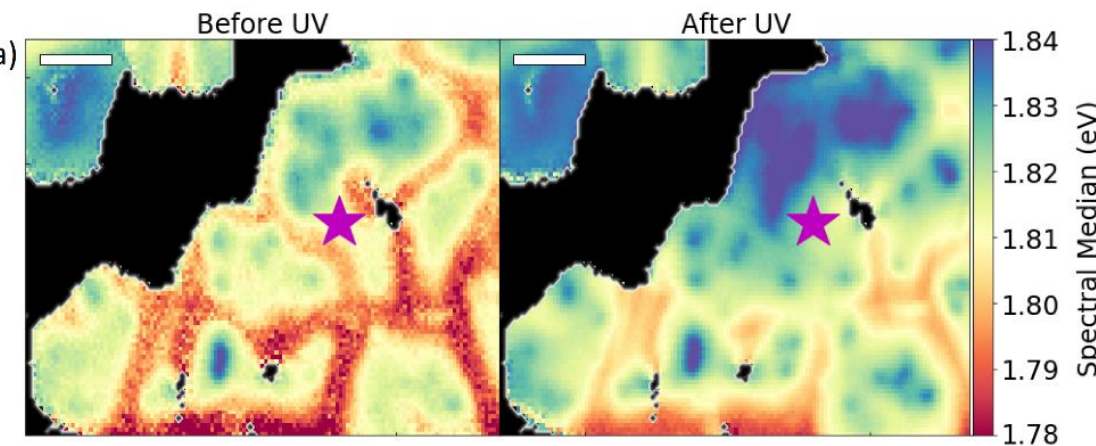

c)

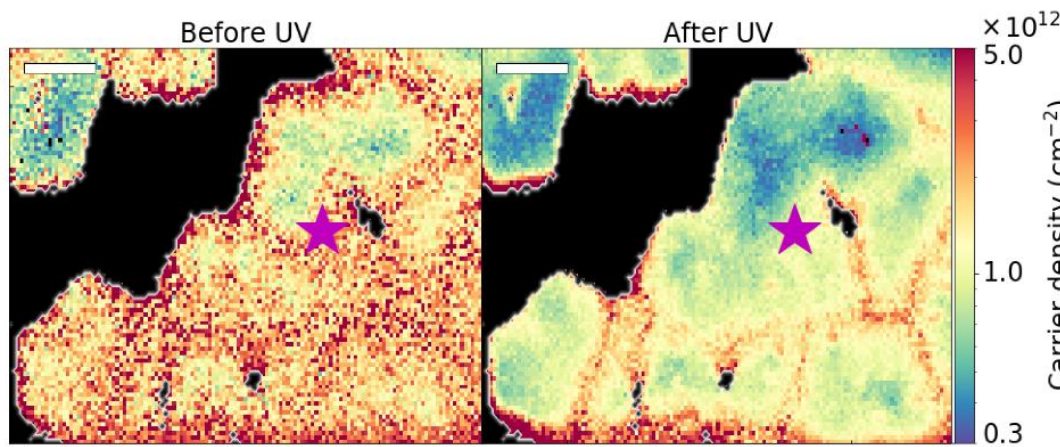

b)

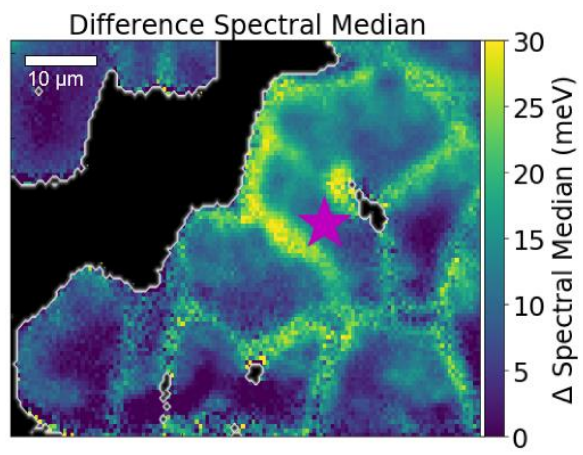

$\Delta$ Charge carrier density

d)

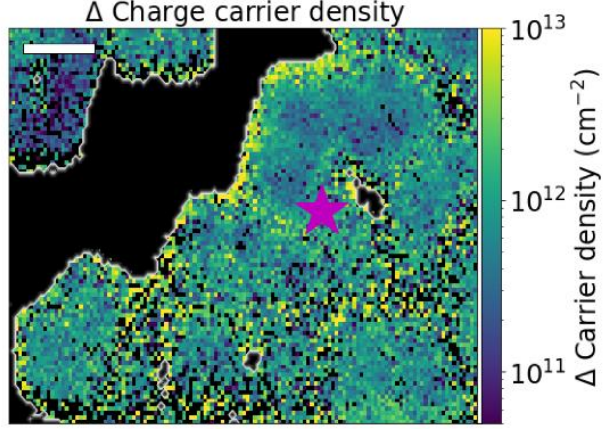

Figure S 6 Spectral map of larger area of monolayer $\mathbf{M o S}_{2}$ flakes merged to each other. Variations in spectral median a) before, b) after photodoping and c) the difference map. Regions characterized with a low PL energy emission are correlated to an higher increase in the spectral median while regions with an initially high PL energy emission experience a less intense blue-shift of the spectral median. Illustration of the a) initial carrier density, of the b) charge density after photodoping and c) the difference map in log scale. The final map presents an undoped area of more than $1210 \mu \mathrm{m}^{2}$ with a carrier density $\mathrm{n}<10^{11} \mathrm{~cm}^{-12}$; initially, the same undoped region had an extension of only $350 \mu \mathrm{m}^{2}$. The average carrier concentration in the $1 \mathrm{~L}-\mathrm{MoS}_{2}$ is suppressed from an initial value of $2.6 \times 10^{12} \mathrm{~cm}^{-12}$ to an average of $1.3 \times 10^{12} \mathrm{~cm}^{-12}$ after the photodoping, due to the injection of photo-holes from ITO nanocrystals. Scale bar $=10 \mu \mathrm{m}$. 

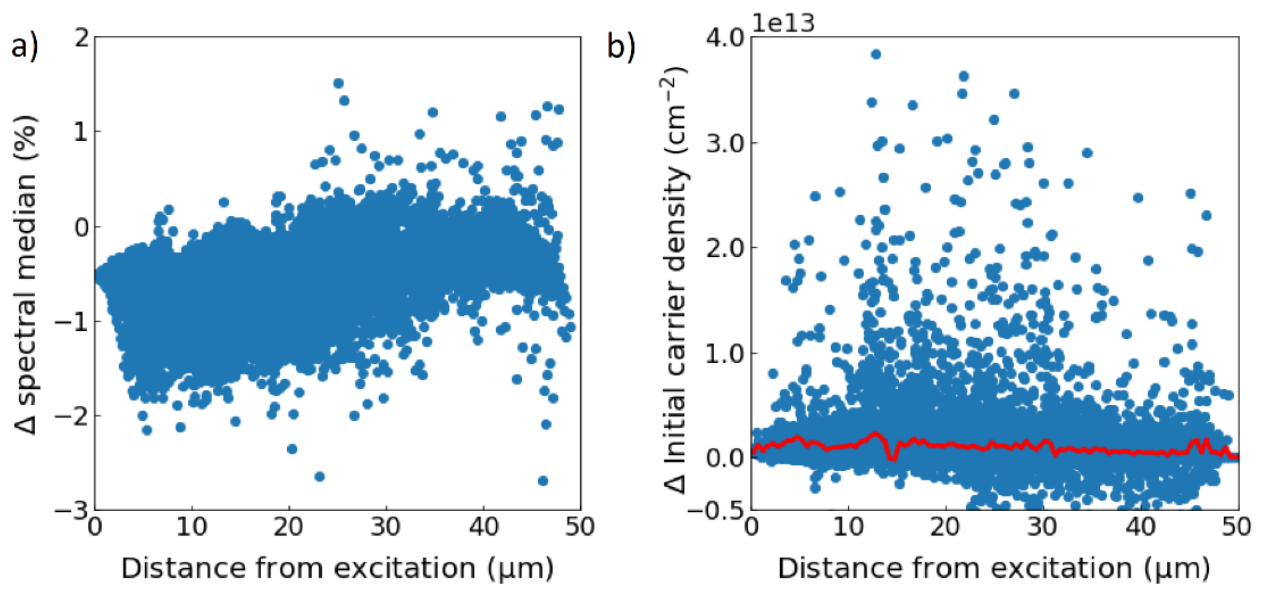

Figure S 7 Photodoping as a function of the distance from the laser spot. Percentage variations (\%) in the spectral median (a) and variations in the initial carrier density (b) through the entire region of the MoS2/ITO hybrid reported in Figure 4 as a function of the distance from the excitation source. We did not find evidence of any strong correlation between photo-induced effects and the distance from the injection spot. Significant changes due to the UV exposure into the MoS2 monolayer are detectable at more than $40 \mu \mathrm{m}$ of distance. 
a)
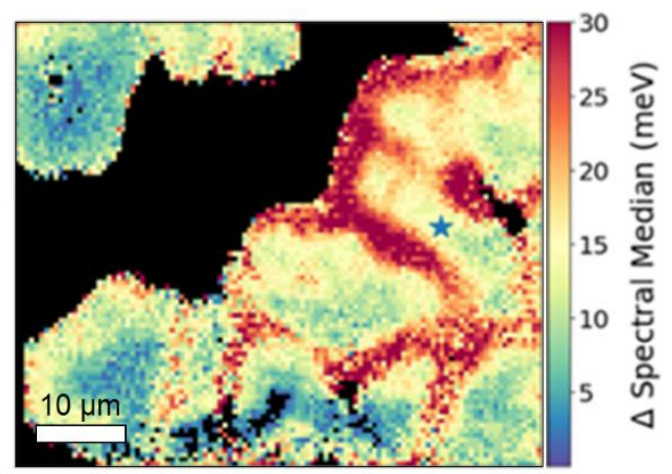

b)

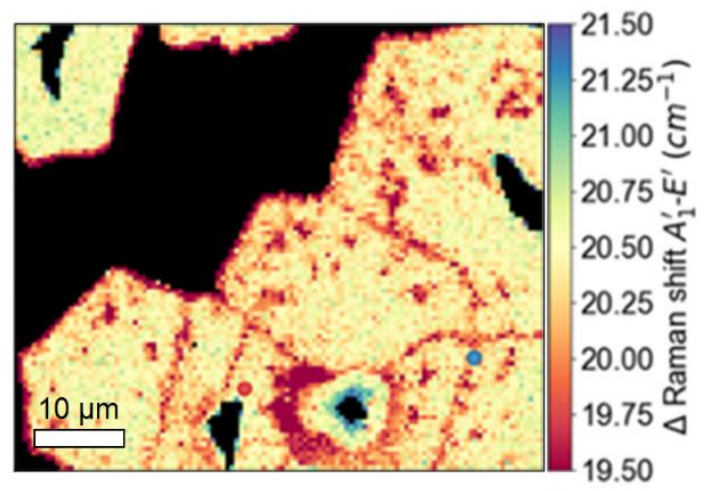

C)

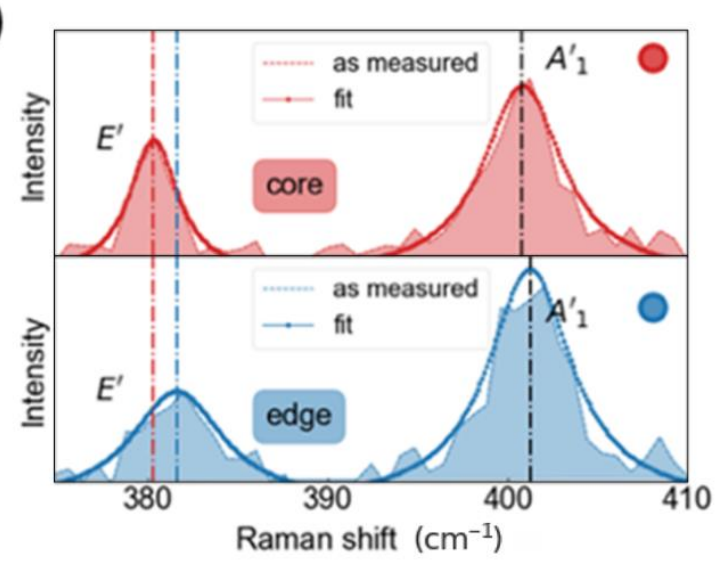

Figure S 8 Raman measurements illustrating relaxed strain along grain boundaries. A measure of strain in monolayer MoS ${ }_{2}$ is the Raman shift of the $\mathrm{E}^{\prime}$ and $\mathrm{A}_{1}$ '. Lower $\Delta$ Raman shifts of $\mathrm{E}^{\prime}$ and $\mathrm{A}_{1}$ ' indicate relaxed tensile strain. In fact, in our Raman map of the same area of investigation grain boundaries of the individual $\mathrm{MoS}_{2}$ flakes are highlighted by lower $\Delta$ Raman shifts, i.e. relaxed tensile strain, and coincide with the largest photo-induced electron extraction. a) The difference map of the spectral median of a larger area of $\mathrm{MoS}_{2}$ flakes illustrating variations of the spectral median of the PL of more than $30 \mathrm{meV}$. Photodoping was induced at the blue colored star in a diffraction limited spot. b) $\Delta$ Raman map of the difference of $\mathrm{A}_{1}$ ' and $\mathrm{E}$ ' modes showing lowest $\Delta$ Raman shift along the grain boundaries, correlated to regions with decreased strain. c) Example of two Raman spectra in the core (red) and edge (blue) region, illustrating that only the E' mode is affected by strain. Spectra correspond to the red and blue dots in Figure 4c. 